\title{
EQUIVARIANT BUNDLE GERBES
}

\author{
MICHAEL K. MURRAY, DAVID MICHAEL ROBERTS, DANNY STEVENSON, \\ AND RAYMOND F. VOZZO
}

\begin{abstract}
We develop the theory of simplicial extensions for bundle gerbes and their characteristic classes with a view towards studying descent problems and equivariance for bundle gerbes. Equivariant bundle gerbes are important in the study of orbifold sigma models. We consider in detail two examples: the basic bundle gerbe on a unitary group and a string structure for a principal bundle. We show that the basic bundle gerbe is equivariant for the conjugation action and calculate its characteristic class; we show also that a string structure gives rise to a bundle gerbe which is equivariant for a natural action of the String 2-group.
\end{abstract}

\section{Contents}

1. Introduction

1.1. Equivariance and simplicial extensions

1.2. 2-group actions

1.3. Summary

2. Background on simplicial manifolds and bundle gerbes

2.1. Simplicial manifolds

2.2. Bundle gerbes and simplicial manifolds

2.3. Simplicial de Rham cohomology

3. Simplicial extensions

3.1. Simplicial extensions of bundle gerbes

3.2. Descent for bundle gerbes

4. Equivariant bundle gerbes

4.2. Descent for equivariant bundle gerbes

4.3. The class of a strongly equivariant bundle gerbe 16

5. The basic bundle gerbe

6. String structures and simplicial extensions 20

6.1. Crossed modules 20

6.2. Bundle 2-gerbes and trivialisations 23

6.3. The Chern-Simons bundle 2-gerbe 24

6.4. The simplicial extension of a string structure 26

Appendix A. Descent for trivialisations 20]

Appendix B. Calculations supporting the proof of Proposition 5.2

References

\section{INTRODUCTION}

Bundle gerbes were introduced by Murray in 32 as a simpler alternative to the gerbes with band $U(1)$ described in the monograph [6] of Brylinski. Bundle gerbes on a manifold

2010 Mathematics Subject Classification. 18G30, 55R91, 53C80.

This research was supported under Australian Research Council's Discovery Projects funding scheme (project numbers DP120100106 and DP130102578). 
$M$ are generalizations of the notion of line bundle on $M$ : this fact is manifested in several ways, one of which is the existence of a characteristic class in $H^{3}(M, \mathbb{Z})$, generalizing the Chern class of a line bundle. In another direction, the notion of bundle gerbe allows for a particularly simple theory of connections and curving leading to a differential form representative for this characteristic class, the 3-curvature of the bundle gerbe connection and curving. Crucially for applications to physics, bundle gerbes give rise to a notion of holonomy over a closed surface, generalizing the notion of the holonomy of a line bundle with connection around a loop.

There are many key examples of bundle gerbes with their origin in physical problems; for example in the study of anomalies in quantum field theory [9, 10, 23]; (together with the allied notion of bundle gerbe module) in the study of $D$-brane charges in string theory [5, 24, 25, 30, 31]; in the study of Chern-Simons theory [11, 29] and its relation to string structures [41; and recently in the study of topological insulators [12, 19. The example which serves to motivate this paper is the role that bundle gerbes and bundle gerbe connections play in $2 \mathrm{D}$ sigma models with a Wess-Zumino term in the action functional. The fields in such a theory are taken to be maps $\phi: \Sigma \rightarrow M$, where $M$ is the target manifold of the theory which is equipped with a closed 3-form $H$. Locally, the Wess-Zumino term $S_{W Z}(\phi)$ is described by integrals over $\Sigma$ of $\phi^{*} B$, where $B$ is a local 2 -form on $M$ solving the equation $d B=H$-the so-called $B$-field. In topologically nontrivial situations there are ambiguities which arise from the various choices that must be made in such a construction. These ambiguities were analyzed by Gawedzki in [18, using the theory of Deligne cohomology, a certain hybrid of Čech and de Rham cohomology. If one interprets the closed 3-form $H$ as the field strength or 3-curvature of a bundle gerbe with connection on $M$, this analysis can be carried out much more systematically and succinctly leading to an identification of the Feynmann amplitudes $\exp \left(i S_{W Z}(\phi)\right)$ in terms of the holonomy of this bundle gerbe with connection [10].

This point of view is particularly well-adapted to the study of Wess-Zumino-Witten sigma models in which the target space is a compact Lie group $G$. In particular, when $G$ is simple and simply connected, there is a canonical bundle gerbe with connection on $G$, the so-called basic bundle gerbe on $G$. The case where the target manifold is a nonsimply connected Lie group arising as the quotient of the simply connected cover $\tilde{G}$ by a finite subgroup $\Gamma$ of the center leads naturally to the notion of a $\Gamma$-equivariant gerbe on $\tilde{G}$. More generally, one can consider the notion of a $\Gamma$-equivariant gerbe on a manifold $M$ upon which $\Gamma$ acts; such an equivariant gerbe amounts to the notion of an ordinary gerbe on the orbifold $M / \Gamma$. These equivariant gerbes can be used to give a similar description of the Wess-Zumino term when the target manifold is such an orbifold. A natural question is how to extend this theory beyond the case of orbifolds, to the case where a compact Lie group acts smoothly on the manifold $M$. This is the motivation for the present work which develops the theory of equivariant bundle gerbes; that is, we have a bundle gerbe $\mathcal{G}=(P, Y)$ over a manifold where $Y \rightarrow M$ is a surjective submersion and a Lie group $G$ acts smoothly on the right of $M$, and we want to investigate in what way this group action can be lifted to $\mathcal{G}$. This question has been studied in [7, 13, for bundle gerbes described by local data over an open cover of $M$, as well as in [3, 21 and notably in the general context of higher geometry in 35. Our approach is to be contrasted with abstract approaches using higher categories in that one often wants, for the purposes of geometry and physics, specific manifolds and explicit descriptions of geometric objects (such as differential forms).

1.1. Equivariance and simplicial extensions. A convenient way of studying equivariant bundle gerbes is to use the theory of simplicial manifolds. To see why this is the case, and to motivate our constructions below, consider first the simpler case of an equivariant $U(1)$-bundle $P \rightarrow M$. Then a right $G$ action on $P$ is a family of bundle maps $\phi_{g}: P \rightarrow P$, each covering the action of the corresponding $g \in G$ and satisfying $\phi_{g} \phi_{h}=\phi_{g h}$. Because all our objects are smooth we would like the bundle maps $\phi_{g}$ to 
depend smoothly on $g$ and a simple way to do that is to introduce the manifold $M \times G$ and two maps $d_{0}, d_{1}: M \times G \rightarrow M$ defined by $d_{0}(m, g)=m g$ and $d_{1}(m, g)=m$. Then the bundle $d_{0}^{-1}(P) \otimes d_{1}^{-1}(P)^{*}$ has fibre at $(m, g)$ given by $P_{m g} \otimes P_{m}^{*}$ and the bundle maps $\phi$ can all be combined to give a section of $d_{0}^{-1}(P) \otimes d_{1}^{-1}(P)^{*}$ whose value at $(m, g)$ is $\left(\phi_{g}\right)_{m}(p) \otimes p^{*}$ where $p \in P_{m}$. The condition that $\phi_{g} \phi_{h}=\phi_{g h}$ now becomes an equation on $M \times G^{2}$. Returning to the case of bundle gerbes, it is natural to replace the idea of an isomorphism with a stable isomorphism and then the condition $\phi_{g} \phi_{h}=\phi_{g h}$ may not hold exactly but rather up to a map $c_{g, h}$ between the stable isomorphisms $\phi_{g} \phi_{h}$ and $\phi_{g h}$. In this case there is a coherence condition on the isomorphisms: $c_{g, h} c_{g h, k}=c_{g, h k} c_{h, k}$, which lives over $M \times G^{3}$. The manifolds $M, M \times G, M \times G^{2}, \ldots$ form a simplicial manifold-the nerve of the action groupoid - which we discuss further in Section 2

Now that we are in the setting of simplicial manifolds it becomes natural to generalise the idea above and formulate a notion of simplicial extension. In the simplest form this starts with a simplicial manifold $X_{\bullet}$ and a bundle gerbe $\mathcal{G}=(P, Y)$ over $X_{0}$. The definition of a simplicial extension then mimics the equivariance condition above. We leave the detail for discussion in Section 3 but note here some geometric consequences. Firstly, given a simplicial manifold $X_{\bullet}$ there is an infinite-dimensional space $\left\|X_{\bullet}\right\|$, called the fat geometric realisation of $X_{\bullet}$, which contains a copy of $X_{0}$. Roughly speaking the existence of a simplicial extension is equivalent to the existence of an extension of the bundle gerbe $\mathcal{G}$ from $X_{0}$ to $\left\|X_{\bullet}\right\|$. We do not prove this fact here but it motivates the choice of name. Secondly, we can realise the real cohomology of $\left\|X_{\bullet}\right\|$ in terms of de Rham classes on the various $X_{k}$ and this is denoted by $H^{n}\left(X_{\bullet}, \mathbb{R}\right)$. There is a natural map

$$
H^{n}\left(X_{\bullet}, \mathbb{R}\right) \rightarrow H^{n}\left(X_{0}, \mathbb{R}\right)
$$

for every $n \geq 0$ corresponding to the pullback from $\left\|X_{\bullet}\right\|$ to $X_{0}$. A simplicial extension of $\mathcal{G}$ defines a class in $H^{3}\left(X_{\bullet}, \mathbb{R}\right)$, which we call the extension class of the simplicial extension, and this maps to the real Dixmier-Douady class of the bundle gerbe $\mathcal{G}$ in $H^{3}\left(X_{0}, \mathbb{R}\right)$.

By working with simplicial manifolds we can also consider the descent problem for bundle gerbes. This has been considered for bundle gerbes described by local data on an open cover of a manifold in [28, 36. If $M \rightarrow N$ is a surjective submersion and $\mathcal{G}$ a bundle gerbe on $M$ then the existence of descent data for $\mathcal{G}$ is precisely the condition for $\mathcal{G}$ to descend to a bundle gerbe on $N$. We show in Section 3 that such descent data is exactly a simplicial extension for the natural simplicial manifold $M, M^{[2]}, M^{[3]}, \ldots$, where $M^{[k]}$ is the $k^{\text {th }}$ fibre product of $M$ with itself over $N$. This result is of interest in its own right but also important in understanding the descent of equivariant bundle gerbes when the action of $G$ on $M$ arises from a principal $G$-bundle $M \rightarrow N$. There are two natural notions of group action on a bundle gerbe; there is a strong action [22, 27, 28, where the group action on $M$ lifts to $Y \rightarrow M$ and also to $P \rightarrow Y^{[2]}$ and commutes with the bundle gerbe product; there is also the notion of weak action, which corresponds to the general simplicial extension setting where essentially the group acts on $\mathcal{G}$ by stable isomorphisms. In Section 4 we show that for both strong and weak $G$ actions on a bundle gerbe $\mathcal{G}$ over the total space of a principal $G$-bundle $M \rightarrow N$ there is a natural notion of quotient or descended gerbe on $N$. In addition we show that a strong action induces a weak action and that the corresponding quotients agree, up to a specified stable isomorphism.

In 34 two of the authors gave a construction of the basic bundle gerbe $\mathcal{B}_{n}$ on a unitary group $U(n)$. In that work we discussed the fact that the conjugation action on $U(n)$ lifted to a strong action of $U(n)$ on $\mathcal{B}_{n}$. In Theorem 5.2 we construct the extension class of this action and note that, in particular, it is non-trivial even in the case of $U(1)$ where $\mathcal{B}_{1}$ and the conjugation action are both trivial. 
1.2. 2-group actions. We also consider the case of an action of a 2-group on a manifold. This statement will need some unpacking. Firstly, a 2 -group ${ }^{1}$ is a monoidal groupoid such that for each object of the groupoid there is another object that is an inverse, possibly only up to isomorphism. For the purposes of this article we will only introduce strict 2 -groups, where associativity holds, inverses are honest inverses and so on. This allows us to use the equivalent but less complicated crossed modules. Also, we are interested in using not just bare groupoids, but Lie groupoids, and so Lie 2-groups. Many known Lie 2-groups, and the ones used in this article, arise as 2-group extensions of ordinary Lie groups. In our case, we take a Lie group $G$ with certain properties, and consider the String 2-group, which fits into an extension

$$
\mathbf{B} U(1) \rightarrow \text { String }_{G} \rightarrow G
$$

for a certain uncomplicated 2-group $\mathbf{B} U(1)$. A lift of the structure group of a principal $G$-bundle $P$ to the group $\operatorname{String}_{G}$ is called a string structure on $P$. These were first considered by Killingback in the context of heterotic string theory in [26] (see also [33, 42 ). The topology and geometry of string structures is also important in Witten's famous paper on the Dirac operator on loop spaces [43] and in Stolz and Teichner's program on elliptic cohomology [39].

It is not difficult to ask for an action of a 2-group on a manifold (all 2-groups will be Lie 2-groups from now on) and it follows from the definition that such an action for String $_{G}$ factors through the map to $G$. The reverse also holds: given a $G$ action, we can induce an action of String $_{G}$.

This, then, is the context in which we look at bundle gerbes that are equivariant under the action of the 2-group $\operatorname{String}_{G}$ on a manifold. While the action factors through $G$, and so may appear uninteresting, an analogue of the discussion above for ordinary group actions becomes much more complicated; here the use of simplicial manifolds and simplicial extensions comes into its own. We shall leave the details for Section 6 , but what we do is consider a string structure for a principal $G$-bundle $P$, which can be given by a bundle gerbe on $P$ and some extra data. This bundle gerbe is not $G$-equivariant, but it is String $_{G}$-equivariant as we shall see in Theorem 6.10.

1.3. Summary. We start in Section 2 with a review of bundle gerbes and various simplicial objects that we need in our subsequent discussion. In Section 3 we present the general definition of our basic notion of a simplicial extension of a bundle gerbe. We present a number of examples and define the simplicial class of a simplicial extension. Our first application uses the notion of simplicial extension to define a general descent condition for bundle gerbes $\mathcal{G}$ over $M$ where $M \rightarrow N$ is a surjective submersion. Our second application of simplicial extensions in Section 4 is to define the notion of weak group action on a bundle gerbe. We show how it relates to the more obvious concept of strong group action and use the idea of descent to define the quotient of a bundle gerbe by a strong or weak group action. We also define equivariant classes for strong and weak group actions. In Section 5 we consider the basic bundle gerbe on a unitary group defined by the first and third authors in 34] and show that it is strongly equivariant under the conjugation action of $U(n)$ on itself. We give an equivariant connective structure and use this to calculate its strongly equivariant class, which is non-trivial even in the case of $U(1)$. Section 6 starts with some preliminary material on crossed modules and bundle 2 -gerbes. We then show that a string structure for a principal $G$-bundle $P$, viewed as a trivialisation of the Chern-Simons bundle 2-gerbe of $P$, gives rise to a natural simplicial extension, meaning that it is equivariant for the natural action of $\operatorname{String}_{G}$ on $P$.

\section{BACKGROUND ON SIMPLICIAL MANIFOLDS AND BUNDLE GERBES}

2.1. Simplicial manifolds. We recall some facts about simplicial objects in a category $\mathscr{C}$ (see for example [4, 14, 20]). We will mostly be interested in the category of smooth

\footnotetext{
${ }^{1}$ not a $p$-group for $p=2$ !
} 
manifolds. Let $\triangle$ be the simplex category, whose objects are the finite ordinal sets $[0]=\{0\},[1]=\{0,1\}, \ldots$ and whose morphisms are order-preserving maps. A simplicial object in $\mathscr{C}$ is a contravariant functor from $\triangle$ to $\mathscr{C}$. A morphism between two simplicial objects is a natural transformation between the two functors defining them.

In more concrete terms, for the category of smooth manifolds, a simplicial object (i.e. a simplicial manifold) is a sequence of manifolds $X_{0}, X_{1}, X_{2}, X_{3}, \ldots$ together with maps $\alpha^{*}: X_{j} \rightarrow X_{i}$ for every arrow $\alpha:[i] \rightarrow[j]$ in $\triangle$, satisfying the compatibility condition $\beta^{*} \alpha^{*}=(\alpha \beta)^{*}$. It is a standard fact that these can all be written in terms of a certain collection of maps $d_{i}: X_{p} \rightarrow X_{p-1}(i=0, \ldots p)$ and $s_{i}: X_{p} \rightarrow X_{p+1}(i=0, \ldots p)$ called face and degeneracy maps, respectively, and satisfying the so-called simplicial identities (see [14). Sometimes, only the face maps of a simplicial object will be important for us and we can ignore the degeneracies. In such a case we will speak of a semi-simplicial object, eg. a semi-simplicial manifold. The face map $d_{k}: X_{p} \rightarrow X_{p-1}$ corresponds to the map $[p-1] \rightarrow[p]$ whose image does not contain $k$. We will typically denote a simplicial manifold $X_{0}, X_{1}, X_{2}, \ldots$ by $X_{\bullet}$. A morphism of simplicial manifolds $Y_{\bullet} \rightarrow X_{\bullet}$ consists of a sequence of maps $Y_{k} \rightarrow X_{k}$ commuting with the face and degeneracy maps.

The following examples will be useful throughout the paper.

Example 2.1. Let $X$ be a manifold. We define $X^{(\bullet)}$ to be the constant simplicial manifold with all face and degeneracy maps equal to the identity. Notice that if $X_{\bullet}$ is a simplicial manifold, then there is a map $X_{0} \rightarrow X_{k}$ corresponding to the unique map $[k] \rightarrow[0]$ and this gives rise to a simplicial map $X_{0}^{(\bullet)} \rightarrow X_{\bullet}$.

Example 2.2. Let $X$ be a manifold. Define $X^{\bullet+1}$ by $X^{k+1}=\operatorname{Map}([k], X)$, with the simplicial maps $X^{i} \rightarrow X^{j}$ given by pullback by $[j] \rightarrow[i]$. Notice that $X^{k+1}$ is the cartesian product of $X$ and the face maps are given by omitting factors.

Example 2.3. Let $Y \rightarrow X$ be a submersion and let $Y^{[k]}$ be the fibre product of $k$ copies of $Y$. This defines a simplicial manifold $Y^{[\bullet+1]}$, where the simplicial maps are induced by restricting those of the cartesian product $Y^{\bullet+1}$.

Example 2.4. If $M$ is a manifold on which a Lie group $G$ acts smoothly we define a simplicial manifold $E G(M)$. by $E G(M)_{n}=M \times G^{n}$ for $n \geq 0$. The face maps are:

$$
d_{k}\left(m, g_{1}, \ldots, g_{n}\right)= \begin{cases}\left(m g_{1}, g_{2}, \ldots, g_{n}\right) & k=0 \\ \left(m, g_{1}, \ldots, g_{k} g_{k+1}, \ldots, g_{n}\right) & k=1,2, \ldots, n-1 \\ \left(m, g_{1}, \ldots, g_{n-1}\right) & k=n .\end{cases}
$$

In particular $X_{1}=M \times G$ and $X_{0}=M$ and the two face maps $X_{1} \rightarrow X_{0}$ are $d_{0}(m, g)=$ $m g$ and $d_{1}(m, g)=m$.

In the case that $M \rightarrow N$ is a principal $G$-bundle then the simplicial manifolds in Examples 2.3 and 2.4 are isomorphic:

Lemma 2.1. If $M \rightarrow N$ is a $G$-bundle then $E G(M) \bullet \simeq M^{[\bullet+1]}$.

Proof. The isomorphism is given by maps $E G(M)_{n} \rightarrow M^{[n]}$ by

$$
\left(m, g_{1}, g_{2}, \ldots, g_{n}\right) \mapsto\left(m, m g_{1}, m g_{1} g_{2}, \ldots, m g_{1} g_{2} \ldots g_{n}\right) .
$$

It can be easily checked that this defines a simplicial map.

Example 2.5. If $M$ is a manifold and $\mathcal{K}$ is a crossed module (Definition 6.1) that acts on $M$ (Definition 6.2), then there is a simplicial manifold $E \mathcal{K}(M)$. similar to the one defined in Example 2.4 for a Lie group. We will use this simplicial manifold in Section 6. where we will give a precise definition.

We call a simplicial object in the category of surjective submersions a simplicial surjective submersion. Explicitly, this will be a pair of simplicial manifolds $Y_{\bullet}$ and $X_{\bullet}$ and 
a simplicial map $Y_{\bullet} \rightarrow X_{\bullet}$ with the property that $Y_{k} \rightarrow X_{k}$ is a surjective submersion for all $k \geq 0$. Note that simplicial surjective submersions are preserved under pullback, in the sense that if $Y_{\bullet} \rightarrow X_{\bullet}$ is a simplicial surjective submersion, then so is the induced map $Y_{\bullet} \times_{X_{\bullet}} Z_{\bullet} \rightarrow Z_{\bullet}$ for any simplicial map $Z_{\bullet} \rightarrow X_{\bullet}$.

Let $X_{\bullet}$ be a simplicial manifold and $Y \rightarrow X_{k}$ a surjective submersion. Define $\delta(Y) \rightarrow$ $X_{k+1}$ by

$$
\delta(Y)=d_{0}^{-1}(Y) \times_{X_{k+1}} d_{1}^{-1}(Y) \times_{X_{k+1}} d_{2}^{-1}(Y) \times_{X_{k+1}} \cdots
$$

Denote by $d_{i}: \delta(Y) \rightarrow Y$ the obvious projections covering the face maps $d_{i}: X_{k+1} \rightarrow X_{k}$. We can define inductively a family $\delta^{k}(Y)$ with maps $\delta^{k}(Y) \rightarrow \delta^{k-1}(Y)$. We remark that $\delta^{\bullet+1}(Y)$ is not a simplicial manifold. However, in the next example we discuss a related construction, which is a simplicial surjective submersion.

Example 2.6. If $X_{\bullet}$ is a simplicial manifold then for each $i=0, \ldots, p$ there are maps $\mu_{i}: X_{p} \rightarrow X_{0}$ induced by the inclusion $[0] \rightarrow[p]$ defined by $0 \mapsto i$. Then

$$
\mu=\left(\mu_{0}, \ldots, \mu_{k}\right): X_{k} \rightarrow X_{0}^{k+1}
$$

defines a morphism of simplicial manifolds $\mu: X_{\bullet} \rightarrow X_{0}^{\bullet+1}$. If $Y \rightarrow X_{0}$ is a surjective submersion then so is $Y^{k+1} \rightarrow X_{0}^{k+1}$ and we can define a simplicial surjective submersion $\mu^{-1}\left(Y^{\bullet+1}\right) \rightarrow X_{\bullet}$. We will use this example extensively in the rest of the paper.

We will use the following similar construction in Section 6 when we discuss bundle 2-gerbes.

Example 2.7. We have maps $\mu_{i j}: X_{p} \rightarrow X_{1}$ for $0 \leq i<j \leq p$ induced by the map $[1] \rightarrow[p]$ defined by $0 \mapsto i$ and $1 \mapsto j$. We can assemble these into maps $X_{k} \rightarrow X_{1}^{k(k+1) / 2}$, which we also call $\mu$. If $Y \rightarrow X_{1}$ is a surjective submersion then as above we can pull back $Y^{k(k+1) / 2}$ to give a surjective submersion over $X_{k}$, for $k \geq 1$. So we have a collection of manifolds

$$
\mu^{-1}(Y)_{k}= \begin{cases}X_{0}, & k=0 \\ Y, & k=1 \\ \mu^{-1}\left(Y^{k(k+1) / 2}\right), & k>1\end{cases}
$$

and maps $\mu^{-1}(Y)_{k} \rightarrow \mu^{-1}(Y)_{k-1}$ satisfying the simplicial identities for face maps. Note that unlike Example 2.6. in general $\mu^{-1}(Y)$. is only a semi-simplicial manifold, since we may not have degeneracy maps $\mu^{-1}(Y)_{0}=X_{0} \rightarrow \mu^{-1}(Y)_{1}=Y$. The canonical map $\mu^{-1}(Y) \bullet \rightarrow X_{\bullet}$ is then a semi-simplicial surjective submersion.

It is clear that one could continue this and define a semi-simplicial surjective submersion given a surjective submersion $Y \rightarrow X_{k}$ as in the paragraph preceding Example 2.6. however we will only need these two cases.

Lemma 2.2. Let $Y_{\bullet} \rightarrow X_{\bullet}$ be a simplicial surjective submersion. For any $k$ there is a map of surjective submersions $Y_{k+1} \rightarrow \delta\left(Y_{k}\right)$ covering projections to $X_{k+1}$ such that each composition $Y_{k+1} \rightarrow \delta\left(Y_{k}\right) \rightarrow Y_{k}$ is the corresponding face map $Y_{k+1} \rightarrow Y_{k}$.

Proof. We define $Y_{k+1} \rightarrow \delta\left(Y_{k}\right)$ by $y \mapsto\left(d_{0}(y), d_{1}(y), \cdots\right)$ and the result follows.

For simplicity, throughout this paper we will only work with a restricted notion of morphism between simplicial surjective submersions. Namely, if $Y_{\bullet}^{\prime} \rightarrow X_{\bullet}$ and $Y_{\bullet} \rightarrow X_{\bullet}$ are simplicial surjective submersions then a morphism

$$
\phi_{\bullet}:\left(Y_{\bullet}^{\prime} \rightarrow X_{\bullet}\right) \rightarrow\left(Y_{\bullet} \rightarrow X_{\bullet}\right)
$$


is a family of maps $\phi_{\bullet}: Y_{\bullet}^{\prime} \rightarrow Y_{\bullet}$ such that every diagram

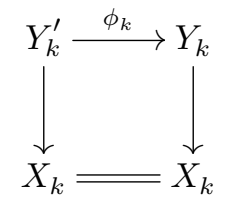

commutes for every $k \geq 0$.

In particular we have

Lemma 2.3. If $Y_{\bullet} \rightarrow X_{\bullet}$ is a simplicial surjective submersion there is a morphism of simplicial surjective submersions

$$
\mu_{\bullet}:\left(Y_{\bullet} \rightarrow X_{\bullet}\right) \rightarrow\left(\mu^{-1}\left(Y_{0}^{\bullet+1}\right) \rightarrow X_{\bullet}\right) .
$$

Proof. For every $k=0,1, \ldots$ we have a commuting diagram

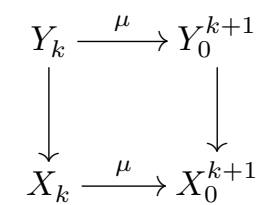

and the result follows from this.

2.2. Bundle gerbes and simplicial manifolds. Let $X$ • be a simplicial manifold and $Q \rightarrow X_{p}$ be a $U(1)$-bundle for some $p \geq 0$. We define a new $U(1)$ bundle $\delta(Q)$ on $X_{p+1}$ by

$$
\delta(Q)=d_{0}^{-1}(Q) \otimes d_{1}^{-1}(Q)^{*} \otimes d_{2}^{-1}(Q) \otimes \cdots
$$

For $i \neq j$ let $\pi_{i j}: X_{p+2} \rightarrow X_{p}$ denote the map induced by the unique order-preserving map $[p] \rightarrow[p+2]$ whose image does not contain $i$ and $j$. Notice that if $i \leq j$ then we have $d_{i} d_{j}=\pi_{i(j+1)}$ and if $i>j$ then $d_{i} d_{j}=\pi_{i j}$. It follows easily that there is an isomorphism

$$
\delta^{2}(Q)=\bigotimes_{0 \leq i<j \leq p+2} \pi_{i j}^{-1}\left(Q \otimes Q^{*}\right)
$$

and hence has $\delta^{2}(Q)$ has a canonical trivialisation. Explicitly, we may define a section $c$ of $\delta^{2}(Q)$ whose value at $x \in X_{p+2}$ is

$$
c(x)=\bigotimes_{0 \leq i<j \leq p+2} \pi_{i j}^{-1}\left(q_{i j} \otimes q_{i j}^{*}\right),
$$

for some choice of elements $q_{i j}$ in the fibres over $\pi_{i j}(x)$. We will usually denote this canonical section by 1 .

Recall the definition of a simplicial line bundle from 8 .

Definition 2.4 ([8]). Let $X$. be a simplicial manifold. A simplicial line bundle over $X$. is a pair $(Q, \sigma)$ defined as follows:

(1) $Q \rightarrow X_{1}$ is a $U(1)$-bundle;

(2) $\sigma$ is a section of $\delta(Q) \rightarrow X_{2}$ such that $\delta(\sigma)=1 \in \delta^{2}(Q)$.

If $Y \rightarrow M$ is a surjective submersion there is an equivalence between bundle gerbes $\mathcal{G}$ over $M$ and simplicial line bundles over $Y^{[\bullet+1]}$. To see this note that if $P$ is a simplicial line bundle over $Y^{[\bullet+1]}$ then $P \rightarrow Y^{[2]}$ is a $U(1)$-bundle and $\sigma\left(y_{1}, y_{2}, y_{3}\right)$ is an element of

$$
P_{\left(y_{2}, y_{3}\right)} \otimes P_{\left(y_{1}, y_{3}\right)}^{*} \otimes P_{\left(y_{1}, y_{2}\right)}
$$

which must be of the form $b \otimes m(a, b)^{*} \otimes a$ for some bundle morphism

$$
m: P_{\left(y_{1}, y_{2}\right)} \otimes P_{\left(y_{2}, y_{3}\right)} \rightarrow P_{\left(y_{1}, y_{3}\right)}
$$

and any elements $a \in P_{\left(y_{1}, y_{2}\right)}$ and $b \in P_{\left(y_{2}, y_{3}\right)}$. The morphism $m$ defines an associative bundle gerbe multiplication if and only if $\delta(\sigma)=1$. If $Y \rightarrow M$ is a surjective submersion 
then we denote the bundle gerbe given by the simplicial line bundle $P$ over $Y^{[\bullet+1]}$ by $(P, Y)$.

We say that a simplicial line bundle $(Q, \sigma)$ over $X_{\bullet}$ is trivial if there is a $U(1)$-bundle $T \rightarrow X_{0}$ such that $Q=\delta(T)$ and $\sigma$ is the section 1 of $\delta^{2}(T)$. It is easy to see that a bundle gerbe is trivial if and only if it is trivial when regarded as a simplicial line bundle. In this case we have a line bundle $T \rightarrow Y$ such that $\delta(T)=P$, where here the $\delta$ operation is for the simplicial manifold $Y^{[\bullet+1]}$. For clarity, we will write $\delta_{Y}(T)$ for this whenever there is any possibility of confusion.

Recall that if $(P, Y)$ is a bundle gerbe on $M$ with trivialisations $T$ and $R$, then there is a canonical definition of a line bundle $L \rightarrow M$ with the property that $T=R \otimes \pi^{*}(L)$. The point is that we have an isomorphism $\delta(T) \rightarrow \delta(R)$ and hence descent data for $T \otimes R^{*}$. We denote the descended line bundle $L$ by $T \oslash R$.

We will also be interested in a particular class of trivial bundle gerbes: we say a bundle gerbe $(P, Y)$ over $M$ is strongly trivial if $P=Y^{[2]} \times U(1)$ and the multiplication is the trivial multiplication, in other words $\left(y_{1}, y_{2}, z_{1}\right)\left(y_{2}, y_{3}, z_{2}\right)=\left(y_{1}, y_{3}, z_{1} z_{2}\right)$. Note that $Y \times U(1)$ is a trivialisation of a strongly trivial bundle gerbe and it follows that if $T$ is a trivialisation of a strongly trivial bundle gerbe then $T=T \oslash(Y \times U(1))$ descends to $M$. If $T$ is trivialised so that $T=Y \times U(1)$ we say that $T$ is a strong trivialisation if the induced trivialisation morphism $\delta_{Y}(Y \times U(1)) \rightarrow Y^{[2]} \times U(1)$ is the identity on the $U(1)$ factor. We have the following Lemma whose proof is straightforward.

Lemma 2.5. Assume that $(Q, Y)$ is strongly trivial over $M$ and trivialised by $T$ so that $T$ descends to $T \oslash(Y \times U(1)) \rightarrow M$ as discussed above. Assume further that $s: Y \rightarrow T$ is a section so it induces an isomorphism $T \simeq Y \times U(1)$. Then $s$ descends to a section of $T \oslash(Y \times U(1)) \rightarrow M$ if and only if the isomorphism $T \simeq Y \times U(1)$ induces a strong trivialisation of $(Q, Y)$.

Let $(P, Y)$ be a bundle gerbe over $M$. If $f: N \rightarrow M$ is a map then the bundle gerbe pulls back to a bundle gerbe $\left(f^{-1}(P), f^{-1}(Y)\right)$ over $N$. If $X \rightarrow M$ is also a submersion and $f: X \rightarrow Y$ is a map of manifolds over $M$ then $\left(f^{-1}(P), X\right)$ is a bundle gerbe over $M$. Notice that both of these examples can be understood as the pullback of the simplicial line bundle over $Y^{[\bullet+1]}$ by $f: f^{-1}\left(Y^{[\bullet+1]}\right) \rightarrow Y^{[\bullet+1]}$ and $f: X^{[\bullet+1]} \rightarrow Y^{[\bullet+1]}$, respectively.

If $T \rightarrow Y$ is a trivialisation of $(P, Y)$ then we denote the induced trivialisation of $\left(f^{-1}(P), f^{-1}(Y)\right)$ by $f^{-1}(T)$.

Assume that $P$ has a bundle gerbe connection $\nabla$ and curving $f$. Choose a connection $\nabla_{T}$ for $T$ satisfying $\nabla=\delta_{Y}\left(\nabla_{T}\right)$. Then $F_{\nabla}=\delta_{Y}\left(F_{\nabla_{T}}\right)=\delta_{Y}(f)$ so that $F_{\nabla_{T}}-f=\pi^{*} \nu_{T}$ for some $\nu_{T} \in \Omega^{2}(M)$. If $R$ is another trivialisation with connection $\nabla_{R}$ such that $\nabla=\delta_{Y}\left(\nabla_{R}\right)$ then, as above, $T \otimes R^{*}$ descends to a bundle $T \oslash R$. Then $\nabla_{T}-\nabla_{R}$ descends to a connection $\nabla_{T \oslash R}$ on $T \oslash R$ whose curvature $F_{T \oslash R}=\nu_{T}-\nu_{R}$.

If $X_{\bullet}$ is a simplicial manifold and $(P, Y)$ a bundle gerbe over $X_{k}$, where $Y \rightarrow X_{k}$ is a surjective submersion, we can define a bundle gerbe $(\delta(P), \delta(Y))$ over $X_{k+1}$. We will also be interested in a more complicated case. Let $Y_{\bullet} \rightarrow X_{\bullet}$ be a simplicial surjective submersion and let $\left(P, Y_{k}\right)$ be a bundle gerbe over $X_{k}$. Then by Lemma 2.2 we can restrict $\left(P, \delta\left(Y_{k}\right)\right)$ to form $\left(P, Y_{k+1}\right)$ over $X_{k+1}$. Further, we can repeat this process and form $\left(\delta^{2}(P), Y_{k+2}\right)$ over $X_{k+2}$. Notice that, as per 2.1), we have that

$$
\delta^{2}(P)=\bigotimes_{0 \leq i<j \leq p+4} \pi_{i j}^{-1}\left(P \otimes P^{*}\right),
$$

where we have used $\pi_{i j}$ to denote the induced map $Y_{k+2}^{[2]} \rightarrow Y_{k}^{[2]}$. Therefore we see that $\left(\delta^{2}(P), Y_{k+2}\right)$ is canonically isomorphic to the strongly trivial bundle gerbe $\left(Y_{k+2}^{[2]} \times\right.$ $\left.U(1), Y_{k+2}\right)$.

We have 
Lemma 2.6. Assume that $\left(Q, Y_{k}\right)$ is a bundle gerbe over $X_{k}$ and $R \rightarrow Y_{k}$ is a trivialisation of $Q$. Then $\left(\delta^{2}(Q), Y_{k+2}\right)$ is strongly trivialised by $\delta^{2}(R) \rightarrow Y_{k+2}$.

Proof. We have that

$$
\delta^{2}(Q)=\bigotimes_{0 \leq i, j, \leq k+4} \pi_{i j}^{-1}(Q) \otimes \pi_{i j}^{-1}\left(Q^{*}\right) \rightarrow Y_{k+2}^{[2]}
$$

and

$$
\delta^{2}(R)=\bigotimes_{0 \leq i, j, \leq k+4} \pi_{i j}^{-1}(R) \otimes \pi_{i j}^{-1}\left(R^{*}\right) \rightarrow Y_{k+2}
$$

If $\psi: \delta_{Y_{k}}(R) \rightarrow Q$ is the trivialisation morphism, the induced trivialisation morphism $\delta_{Y_{k+2}}\left(\delta^{2}(T)\right) \rightarrow \delta^{2}(Q)$ is

$$
\bigotimes_{0 \leq i, j, \leq k+4} \pi_{i j}^{-1}(\psi) \otimes \pi_{i j}^{-1}\left(\psi^{*}\right) \rightarrow Y_{k+2}
$$

which is the trivial morphism induced by the identity map on $U(1)$.

We will be particularly interested in the following examples of this:

Example 2.8. If $Y \rightarrow X_{0}$ is a surjective submersion and $(P, Y)$ is a bundle gerbe, then we have the simplicial surjective submersion $\mu^{-1}\left(Y^{\bullet+1}\right) \rightarrow X$. from Example 2.6 and we can form the bundle gerbe $\left(\delta(P), \mu^{-1}\left(Y^{2}\right)\right)$ over $X_{1}$ and the bundle gerbe $\left(\delta^{2}(P), \mu^{-1}\left(Y^{3}\right)\right)$ over $X_{2}$.

Example 2.9. If $Y \rightarrow X_{1}$ is a surjective submersion and $(P, Y)$ is a bundle gerbe, then we have the semi-simplicial surjective submersion $\mu^{-1}(Y) \bullet \rightarrow X_{\bullet}$ from Example 2.7 and we can form the bundle gerbe $\left(\delta(P), \mu^{-1}(Y)_{2}\right)$ over $X_{2}$ and the bundle gerbe $\left(\delta^{2}(P), \mu^{-1}(Y)_{3}\right)$ over $X_{3}$.

Finally, we make a remark about notation. We will be concerned with bundle gerbes $\mathcal{G}=(P, Y)$ over simplicial manifolds and we shall be using the operation $\delta$ repeatedly. As in Examples 2.8 and 2.9 and the discussion preceding Lemma 2.6 we will often be interested in the bundle gerbe $(\delta(P), \delta(Y))$ restricted to some subspace of $\delta(Y)$. To make it clear precisely which bundle gerbe we mean by $\delta(\mathcal{G})$, we will use the notation $\left(\delta(P), Y_{k}\right)$ (where $Y_{\bullet} \rightarrow X_{\bullet}$ is a simplicial surjective submersion) whenever there is chance of confusion.

2.3. Simplicial de Rham cohomology. We recall the definition of the simplicial de Rham cohomology of a simplicial manifold $X_{\bullet}$ [14]. Associated canonically to $X_{\bullet}$ is the bicomplex with differentials

$$
\begin{aligned}
D_{p, q}: \Omega^{p}\left(X_{q}\right) & \rightarrow \Omega^{p+1}\left(X_{q}\right) \oplus \Omega^{p}\left(X_{q+1}\right) \\
\eta_{(p, q)} & \mapsto\left((-1)^{q} d \eta_{(p, q)}, \delta \eta_{(p, q)}\right) .
\end{aligned}
$$

We combine these to form the total complex in the usual fashion:

$$
D: \bigoplus_{p+q=r} \Omega^{p}\left(X_{q}\right) \rightarrow \bigoplus_{p+q=r+1} \Omega^{p}\left(X_{q}\right)
$$

The cohomology of this total complex is defined to be the simplicial de Rham cohomology, denoted $H^{r}\left(X_{\bullet}, \mathbb{R}\right)$. Note that this is also the real cohomology of the fat realisation $\left\|X_{\bullet}\right\|$ (see for instance Proposition 5.15 of [14]). For later convenience we introduce the notation $\mathcal{A}^{*}\left(X_{\bullet}\right)$ for this total complex.

Of particular interest will be $H^{3}\left(X_{\bullet}, \mathbb{R}\right)$ and we note that a class consists of

$$
\eta=\left(\eta_{(0,3)}, \eta_{(1,2)}, \eta_{(2,1)}, \eta_{(3,0)}\right) \in \Omega^{0}\left(X_{3}\right) \oplus \Omega^{1}\left(X_{2}\right) \oplus \Omega^{2}\left(X_{1}\right) \oplus \Omega^{3}\left(X_{0}\right)
$$


satisfying

$$
\begin{aligned}
D \eta & =\left(\delta \eta_{(0,3)},-d \eta_{(0,3)}+\delta \eta_{(1,2)}, d \eta_{(1,2)}+\delta \eta_{(2,1)},-d \eta_{(2,1)}+\delta \eta_{(3,0)}, d \eta_{(3,0)}\right) \\
& =(0,0,0,0,0)
\end{aligned}
$$

up to addition of a cocycle of the form $D \rho$ so that

$$
\begin{aligned}
\eta+D \rho=\left(\eta_{(0,3)}+\delta \rho_{(0,2)}, \eta_{(1,2)}+d \rho_{(0,2)}+\right. & \delta \rho_{(1,1)}, \\
& \left.\eta_{(2,1)}-d \rho_{(1,1)}+\delta \rho_{(2,0)}, \eta_{(3,0)}+d \rho_{(2,0)}\right) .
\end{aligned}
$$

\section{Simplicial EXtensions}

3.1. Simplicial extensions of bundle gerbes. Before we define the notion of a simplicial extension we need the following:

Proposition 3.1. Let $Y_{\bullet} \rightarrow X_{\bullet}$ be a simplicial surjective submersion and $\mathcal{G}=\left(P, Y_{k}\right)$ be a bundle gerbe over $X_{k}$. Assume that $\delta(\mathcal{G})=\left(\delta(P), Y_{k+1}\right)$ has a trivialisation $T \rightarrow Y_{k+1}$. Then $\delta(T) \rightarrow Y_{k+2}$ descends to a line bundle $A_{T} \rightarrow X_{k+2}$ and the canonical trivialisation of $\delta^{2}(T) \rightarrow Y_{k+3}$ descends to a trivialisation of $\delta\left(A_{T}\right) \rightarrow X_{k+3}$.

Proof. Notice first that the line bundle $\delta(T)$ trivialises the bundle gerbe $\left(\delta^{2}(P), Y_{k+2}\right)$, which is canonically isomorphic to the strongly trivial bundle gerbe $\left(Y_{k+2}^{[2]} \times U(1), Y_{k+2}\right)$, as we observed in the discussion preceding Lemma 2.6. Hence $\delta(T)$ descends to $A_{T}:=$ $\delta(T) \oslash\left(Y_{k+2} \times U(1)\right) \rightarrow X_{k+2}$.

To see that the canonical trivialisation of $\delta^{2}(T) \rightarrow Y_{k+3}$ descends to $\delta\left(A_{T}\right) \rightarrow X_{k+3}$ we apply Lemma 2.6 to the bundle gerbe $\left(\delta(P), Y_{k+1}\right)$ with trivialisation $T \rightarrow Y_{k+1}$ to deduce that $\left(\delta^{3}(P), Y_{k+3}\right)$ is strongly trivialised by $\delta^{2}(T) \rightarrow Y_{k+3}$. Then Lemma 2.5 implies that the section of $\delta^{2}(T)$ descends to a section of $\delta\left(A_{T}\right) \rightarrow X_{k+3}$.

Using this we can make the following definition:

Definition 3.2. Let $X_{\bullet}$ be a simplicial manifold and $\mathcal{G}=\left(P, Y_{0}\right)$ be a bundle gerbe over $X_{0}$. A simplicial extension of $\mathcal{G}$ over $X_{\bullet}$ is a triple $\left(Y_{\bullet}, T, s\right)$ consisting of:

(1) $Y_{\bullet} \rightarrow X_{\bullet}$ a simplicial surjective submersion;

(2) a trivialisation $T \rightarrow Y_{1}$ of $\delta(\mathcal{G})=\left(\delta(P), Y_{1}\right)$ over $X_{1}$; and

(3) a section $s: X_{2} \rightarrow A_{T}$ satisfying $\delta(s)=1$ relative to the canonical trivialisation of $\delta\left(A_{T}\right)$.

If $\left(Y_{\bullet}, T, s\right)$ is a simplicial extension then pulling back $s$ to a section of $\delta(T)$ defines a simplicial line bundle over $Y_{\bullet}$, which we denote by $[T, s]$.

A simple but useful example of a simplicial extension is the following, defined for any bundle gerbe $(P, Y)$ over $X$.

Proposition 3.3. A bundle gerbe $(P, Y)$ over a manifold $X$ defines a simplicial extension $\left(Y^{[\bullet+1]}, T, s\right)$ over the constant simplicial manifold $X^{(\bullet)}$ whose induced simplicial line bundle on $Y^{[\bullet+1]}$ is precisely $(P, Y)$.

Proof. The condition that $T$ trivialises $\delta(P)$ can be written as follows. Let $\left(y_{0}, y_{1}\right)$, $\left(y_{0}^{\prime}, y_{1}^{\prime}\right),\left(y_{0}^{\prime \prime}, y_{1}^{\prime \prime}\right) \in Y^{[2]}$, then there is an isomorphism

$$
T_{\left(y_{0}, y_{1}\right)} \simeq P_{\left(y_{0}, y_{0}^{\prime}\right)} \otimes T_{\left(y_{0}^{\prime}, y_{1}^{\prime}\right)} \otimes P_{\left(y_{1}^{\prime}, y_{1}\right)}
$$

and the isomorphism

$$
T_{\left(y_{0}, y_{1}\right)} \simeq P_{\left(y_{0}, y_{0}^{\prime \prime}\right)} \otimes T_{\left(y_{0}^{\prime \prime}, y_{1}^{\prime \prime}\right)} \otimes P_{\left(y_{1}^{\prime \prime}, y_{1}\right)}
$$

is equal to the induced isomorphism

$$
\begin{aligned}
T_{\left(y_{0}, y_{1}\right)} & \simeq P_{\left(y_{0}, y_{0}^{\prime}\right)} \otimes T_{\left(y_{0}^{\prime}, y_{1}^{\prime}\right)} \otimes P_{\left(y_{1}^{\prime}, y_{1}\right)} \\
& \simeq P_{\left(y_{0}, y_{0}^{\prime}\right)} \otimes P_{\left(y_{0}^{\prime}, y_{0}^{\prime \prime}\right)} \otimes T_{\left(y_{0}^{\prime \prime}, y_{1}^{\prime \prime}\right)} \otimes P_{\left(y_{1}^{\prime \prime}, y_{1}^{\prime}\right)} \otimes P_{\left(y_{1}^{\prime}, y_{1}\right)} \\
& \simeq P_{\left(y_{0}, y_{0}^{\prime \prime}\right)} \otimes T_{\left(y_{0}^{\prime \prime}, y_{1}^{\prime \prime}\right)} \otimes P_{\left(y_{1}^{\prime \prime}, y_{1}\right)},
\end{aligned}
$$


where we use the bundle gerbe multiplication to get from the second to the third line.

In this case, a trivialisation $T \rightarrow Y^{[2]}$ is given by $T_{\left(y_{0}, y_{1}\right)}=P_{\left(y_{0}, y_{1}\right)}$ and defining the map using the bundle gerbe product. Then

$$
\delta(T)_{\left(y_{0}, y_{1}, y_{2}\right)}=P_{\left(y_{1}, y_{2}\right)} \otimes P_{\left(y_{0}, y_{2}\right)}^{*} \otimes P_{\left(y_{0}, y_{1}\right)},
$$

and a section is given by the bundle gerbe multiplication. It is easy to see that the descent equation preserves this section and this defines the required section $s$ of $A_{T}$. The associativity of the bundle gerbe product gives us $\delta(s)=1$. By construction this simplicial extension pulls back to the simplicial line bundle defined by $(P, Y)$.

Proposition 3.4. Let $\left(Y_{\bullet}^{\prime} \rightarrow X_{\bullet}\right)$ and $\left(Y_{\bullet} \rightarrow X_{\bullet}\right)$ be simplicial surjective submersions where $\left(Y_{0}^{\prime} \rightarrow X_{0}\right)=\left(Y_{0} \rightarrow X_{0}\right)$. Assume we have a morphism $\phi:\left(Y_{\bullet}^{\prime} \rightarrow X_{\bullet}\right) \rightarrow\left(Y_{\bullet} \rightarrow\right.$ $\left.X_{\bullet}\right)$ which is the identity when $k=0$. Then a simplicial extension $\left(Y_{\bullet}, T, s\right)$ of $\left(P, Y_{0}\right)$ over $X_{\bullet}$ pulls back to a simplicial extension $\left(Y_{\bullet}^{\prime}, \phi^{-1}(T), s\right)$ of $\left(P, Y_{0}\right)$ over $X_{\bullet}$.

Proof. The trivialisation $T$ of $\left(\delta(P), Y_{1}\right)$ pulls back to a trivialisation $\phi^{-1}(T)$ of the bundle gerbe $\phi^{-1}\left(\delta(P), Y_{1}\right)=\left(\delta(P), Y_{1}^{\prime}\right)$. Further, since $\phi$ is a simplicial map, $\delta\left(\phi^{-1}(T)\right)=$ $\phi^{-1}(\delta(T))$ which descends to $A_{T}$. Hence the triple $\left(Y_{\bullet}^{\prime}, \phi^{-1}(T), s\right)$ is a simplicial extension of $\left(P, Y_{0}\right)$.

More surprising is the following:

Proposition 3.5. Let $\left(Y_{\bullet} \rightarrow X_{\bullet}\right)$ be a simplicial surjective submersion and $\left(Y_{\bullet}, T, s\right)$ be a simplicial extension of $\left(P, Y_{0}\right)$ over $X_{\bullet}$. Then there is a simplicial extension

$$
\left(\mu^{-1}\left(Y_{0}^{\bullet+1}\right), \mu(T), \mu(s)\right)
$$

which pulls back to $\left(Y_{\bullet}, T, s\right)$ by the morphism in Proposition 3.4 .

Proof. Notice first that over $X_{1}$ we have two bundle gerbes $\left(\delta(P), \mu^{-1}\left(Y^{2}\right)\right)$ and its pullback by $\mu: Y_{1} \rightarrow \mu^{-1}\left(Y^{2}\right)$ which is $\left(\mu^{-1}(\delta(P)), Y_{1}\right)$. If $T \rightarrow Y_{1}$ is a trivialisation of $\left(\mu^{-1}(\delta(P)), Y_{1}\right)$ then from Proposition A.1 we know that there is an induced trivialisation $\mu(T) \rightarrow \mu^{-1}\left(Y^{2}\right)$ of $\left(\delta(P), \mu^{-1}\left(Y^{2}\right)\right)$ which pulls back to $T \rightarrow Y_{1}$. The construction of $A_{T}$ in Proposition 3.1 depends only on $\delta(P)$ and $T$ and it follows that there are isomorphisms $A_{T} \simeq A_{\mu(T)}$ which commute with the trivialisations of $\delta\left(A_{T}\right)$ and $\delta\left(A_{\mu(T)}\right)$. Hence we can define a section $\mu(s)$ of $A_{\mu(T)}$ and $\left(\mu^{-1}\left(Y_{0}^{\bullet+1}\right), \mu(T), \mu(s)\right)$ is a simplicial extension. By Proposition A.1 and the construction it pulls back in the required manner.

Proposition 3.5 means we could simplify the definition of simplicial extension by always working with the simplicial surjective submersion $\mu^{-1}\left(Y_{0}^{\bullet+1}\right) \rightarrow X_{\bullet}$. Indeed, when we specify a simplicial extension using only the pair $(T, s)$ then it will be understood that $Y_{\bullet}=\mu^{-1}\left(Y_{0}^{\bullet+1}\right)$. We note however that as we shall see in the next section, in practice we find that it is useful to allow the extra flexibility of the choice of $Y_{\bullet}$.

Recall from Example 2.1 that we have the map $X_{0} \rightarrow X_{k}$ induced by the unique map $[k] \rightarrow[0]$ and that the composition $X_{0} \rightarrow X_{k} \rightarrow X_{0}$ with each of the projections $\mu_{i}: X_{k} \rightarrow X_{0}$ is the identity. This means $\mu^{-1}\left(Y^{\bullet+1}\right) \rightarrow X_{\bullet}$ pulls back to $Y^{[\bullet+1]} \rightarrow X_{0}^{(\bullet)}$ and the simplicial line bundle $[T, s]$ pulls back to a simplicial line bundle on $Y^{[\bullet+1]}$, which is a bundle gerbe on $X_{0}$. We have

Proposition 3.6. Let $(T, s)$ be a simplicial extension of the bundle gerbe $(P, Y)$ over $X_{\bullet}$. The bundle gerbe on $X_{0}$ defined by the pullback of $[T, s]$ to $X_{0}^{(\bullet)}$ via the simplicial map $X_{0}^{(\bullet)} \rightarrow X$ • is isomorphic to $(P, Y)$.

Proof. The pullback of the simplicial extension of $(P, Y)$ over the simplicial manifold $X_{\bullet}$ is a simplicial extension of $(P, Y)$ over $X_{0}^{(\bullet)}$. We know from Proposition 3.3 that $(P, Y)$ also defines a simplicial extension over $X_{0}^{(\bullet)}$. So we only need to prove that any two simplicial extensions of $(P, Y)$ over $X_{0}^{(\bullet)}$ are isomorphic. Assume then that $\left(T_{1}, s_{1}\right)$ 
and $\left(T_{2}, s_{2}\right)$ are simplicial extensions of $(P, Y)$ over $X_{0}^{(\bullet)}$. Notice that $T_{1}$ and $T_{2}$ are trivialisations of $\delta(P)$ so there exists a line bundle $L \rightarrow X_{0}$ defined by $L=T_{1} \oslash T_{2}$. Then $\delta(L)=A_{T_{1}} \otimes A_{T_{2}}^{*}$. But as all the maps are the identity $\delta(L)=L$, which has a section defined by $s_{1} \otimes s_{2}^{*}$. Hence $L$ is trivial which defines an isomorphism from $T_{1}$ to $T_{2}$. Moreover, by definition this isomorphism maps $s_{1}$ to $s_{2}$ and hence defines an isomorphism of simplicial line bundles.

The same canonical simplicial map $X_{0}^{(\bullet)} \rightarrow X_{\bullet}$ induces a homomorphism

$$
H^{n}\left(X_{\bullet}, \mathbb{R}\right) \rightarrow H^{n}\left(X_{0}, \mathbb{R}\right)
$$

for every $n \geq 0$.

Proposition 3.7. A simplicial extension of $\mathcal{G}$ over $X$ • defines a class in the simplicial de Rham cohomology $H^{3}\left(X_{\bullet}, \mathbb{R}\right)$ which maps to the real Dixmier-Douady class of $\mathcal{G}$ in $H^{3}\left(X_{0}, \mathbb{R}\right)$.

Proof. Let the bundle gerbe $\mathcal{G}=\left(P, Y_{0}\right)$ where $\pi: Y_{0} \rightarrow X_{0}$ is a surjective submersion. Let $(\nabla, f)$ be a connection and curving for $\mathcal{G}$ and denote by $\eta_{(3,0)} \in \Omega^{3}\left(X_{0}\right)$ the corresponding three-curvature so that $\pi^{*}\left(\eta_{(3,0)}\right)=d f$. Note that this is unique up to addition to $f$ of $\pi^{*}\left(\rho_{(2,0)}\right)$ where $\rho_{(2,0)} \in \Omega^{2}\left(X_{0}\right)$, which changes $\eta_{(3,0)}$ to $\eta_{(3,0)}+d \rho_{(2,0)}$.

Notice first that $T$ is a trivialisation of $\delta(\mathcal{G})$ over $X_{1}$ and that $\delta(\mathcal{G})$ has connection $\delta(\nabla)$ and curving $\delta(f)$. As in the discussion following Lemma 2.5 we choose a connection $\nabla_{T}$ for $T$ satisfying $\delta_{Y}\left(\nabla_{T}\right)=\delta(\nabla)$ and define $\eta_{(2,1)}=-\nu_{T} \in \Omega^{2}\left(X_{1}\right)$ so that

$$
\pi^{*}\left(\eta_{(2,1)}\right)=-F_{T}+\delta(f) .
$$

Hence $\pi^{*}\left(\delta\left(\eta_{(3,0)}\right)\right)=\delta(d f)=\pi^{*}\left(d \eta_{(2,1)}\right)$ so that $-d \eta_{(2,1)}+\delta\left(\eta_{(3,0)}\right)=0$ as required.

The choice of $\nabla_{T}$ is unique up to adding $\pi^{*}\left(\rho_{(1,1)}\right)$ where $\rho_{(1,1)} \in \Omega^{1}\left(X_{1}\right)$ which changes $\eta_{(2,1)}$ to $\eta_{(2,1)}-d \rho_{(1,1)}$. If we also change $f$ as above then we change $\eta_{(2,1)}$ to $\eta_{(2,1)}-d \rho_{(1,1)}+\delta\left(\rho_{(2,0)}\right)$.

Notice that $\delta(T)$ has a connection $\delta\left(\nabla_{T}\right)$ whose curvature is $\delta\left(\nu_{T}\right)=\delta\left(\eta_{(2,1)}\right)$. This descends to a connection $\nabla_{A_{T}}$ on $A_{T}$ which has a trivialising section $s$. We define

$$
\eta_{(1,2)}=s^{*}\left(\nabla_{A_{T}}\right) \in \Omega^{1}\left(X_{2}\right)
$$

so that

$$
\pi^{*}\left(\eta_{(1,2)}\right)=s^{*}\left(\delta\left(\nabla_{T}\right)\right) \in \Omega^{1}\left(X_{2}\right) .
$$

Moreover $d \pi^{*}\left(\eta_{(1,2)}\right)=-\pi^{*}\left(\delta \eta_{(2,1)}\right)$. Hence $d \eta_{(1,2)}+\delta\left(\eta_{(2,1)}\right)=0$.

Notice that if we change $\nabla_{T}$ by adding $\pi^{*}\left(\rho_{(1,1)}\right)$ then $\eta_{(1,2)}$ changes by addition of $\delta\left(\rho_{(1,1)}\right)$.

Lastly because $\delta(s)=1$ we conclude that $\delta\left(\eta_{(1,2)}\right)=0$.

Notice that if we change $(\nabla, f)$ to $(\nabla+\delta(\alpha), f+d \alpha)$ for $\alpha \in \Omega^{1}\left(Y_{0}\right)$, then $\nabla_{T}$ changes to $\nabla_{T}+\alpha$ and the cocycle is unchanged.

Finally we conclude that the simplicial extension defines a cocycle

$$
\eta=\left(0, \eta_{(1,2)}, \eta_{(2,1)}, \eta_{(3,0)}\right) \in \mathcal{A}^{3}\left(X_{\bullet}\right),
$$

whose class in $H^{3}\left(X_{\bullet}, \mathbb{R}\right)$ we have seen is independent of choices.

We note that

Proposition 3.8. The pullback of simplicial extensions defined in Proposition 3.4 preserves simplicial classes.

Proof. This follows immediately from the construction as all the data used to define the class pulls back.

Definition 3.9. We call the class defined in Proposition 3.7 the (real) extension class of the simplicial extension and denote it by $\epsilon\left(Y_{\bullet}, T, s\right)$, or simply $\epsilon(T, s)$, since the class is independent of $Y_{\bullet}$. 
Remark 3.1. Although we shall not need to make use of them, it is worth pointing out the following facts. To every simplicial extension of $\mathcal{G}$ over $X_{\bullet}$ there is associated an integral extension class in $H^{3}\left(\left\|X_{\bullet}\right\|, \mathbb{Z}\right)$ which classifies simplicial extensions in the sense that there is an isomorphism between a suitable set of equivalence class of simplicial extensions and $H^{3}\left(\left\|X_{\bullet}\right\|, \mathbb{Z}\right)$. Furthermore, a bundle gerbe $\mathcal{G}$ over $X_{0}$ has a simplicial extension if and only if there is a bundle gerbe $\widetilde{\mathcal{G}}$ over the geometric realization $\left\|X_{\bullet}\right\|$ whose DixmierDouady class in $H^{3}(\|X \bullet\|, \mathbb{Z})$ is the integral extension class of the simplicial extension and whose restriction to $X_{0}$ is stably isomorphic to $\mathcal{G}$. A proof of this fact uses some of the machinery of simplicial Cech cohomology (see for example [16]), which would require a lengthy discussion. Since these facts are not central to our paper we have chosen to omit them.

Proposition 3.10. If $\left(T_{i}, s_{i}\right)$ is a simplicial extension of $\mathcal{G}_{i}$ for $i=1,2$ then $\left(T_{1}, s_{1}\right) \otimes$ $\left(T_{2}, s_{2}\right)=\left(T_{1} \otimes T_{2}, s_{1} \otimes s_{2}\right)$ is a simplicial extension for $\mathcal{G}_{1} \otimes \mathcal{G}_{2}$ and $\epsilon\left(\left(T_{1}, s_{1}\right) \otimes\left(T_{2}, s_{2}\right)\right)=$ $\epsilon\left(T_{1}, s_{1}\right)+\epsilon\left(T_{2}, s_{2}\right)$.

When $\mathcal{G}$ is trivial so that $\mathcal{G}=\left(\delta_{Y}(R), Y\right)$ for $R \rightarrow Y$ we can construct a trivial simplicial extension $(\delta(R), 1)$, where $\delta(R)$ denotes the induced trivialisation of $\delta(\mathcal{G})$.

If we regard $X_{0} \rightarrow X_{0}$ as a surjective submersion we can identify $X_{0}^{[2]}$ with $X_{0}$ and the trivial line bundle $X_{0} \times U(1)$ gives us a strongly trivial bundle gerbe. The product of any bundle gerbe $\mathcal{G}$ with this bundle gerbe is naturally isomorphic to itself. Any simplicial line bundle $(J, \sigma)$ gives us a simplicial extension $(J, \sigma)$ of this trivial bundle gerbe.

It follows that a simplicial line bundle can form a product with a simplicial extension to give rise to a new simplicial extension. Or more directly, given a simplicial extension $\left(Y_{\bullet}, T, s\right)$ with $\pi: Y_{\bullet} \rightarrow X_{\bullet}$ and a simplicial line bundle $(J, \sigma)$, we can define a new trivialisation $T \otimes \pi^{-1}(J)$. Then $A_{T \otimes \pi^{-1}(J)}=A_{T} \otimes \delta(J)$ which has a section $s \otimes \delta(\sigma)$. Hence we have a new simplicial extension $(T, s) \otimes(J, \sigma)=\left(T \otimes \pi^{-1}(J), s \otimes \delta(\sigma)\right)$.

If we pick a connection $\nabla_{J}$ for $J$ then we obtain an integral two-form $F_{J} \in \Omega^{2}\left(X_{1}\right)$ with $d F_{J}=0$. Also we can define $\alpha \in \Omega^{1}\left(X_{2}\right)$ by $\alpha=\sigma^{*}\left(\delta\left(\nabla_{J}\right)\right)$ and $\delta(\alpha)=$ $(\delta \sigma)^{*}\left(\delta^{2}\left(\nabla_{J}\right)\right)=0$ and $\delta\left(F_{J}\right)=d \alpha$. Hence a simplicial line bundle has a simplicial Chern class $c_{s}(J, \sigma) \in H^{3}\left(X_{\bullet}, \mathbb{R}\right)$ represented by $(0, \alpha, F, 0)$. Clearly this is in the kernel of the map $H^{3}\left(X_{\bullet}, \mathbb{R}\right) \rightarrow H^{3}\left(X_{0}, \mathbb{R}\right)$.

If $(T, s)$ and $\mathcal{G}$ has the class

$$
\left(0, \eta_{(1,2)}, \eta_{(2,1)}, \eta_{(3,0)}\right)
$$

then $\left(T \otimes \pi^{-1}(J), s \otimes \delta(\sigma)\right)$ has the class

$$
\left(0, \eta_{(1,2)}+\alpha, \eta_{(2,1)}+F, \eta_{(3,0)}\right) .
$$

and hence $\epsilon((T, s) \otimes(J, \sigma))=\epsilon(T, s)+c_{s}(J, \sigma)$. In fact the converse of this is true.

Proposition 3.11. Let $\left(T_{1}, s_{1}\right)$ and $\left(T_{2}, s_{2}\right)$ be two simplicial extensions of $\mathcal{G}$. Then there exists a simplicial line bundle $\left(T_{1} \oslash T_{2}, s_{1} \otimes s_{2}^{*}\right)$ such that $\left(T_{1}, s_{2}\right)=\left(T_{2}, s_{2}\right) \otimes\left(T_{1} \oslash\right.$ $\left.T_{2}, s_{1} \otimes s_{2}^{*}\right)$.

Proof. The construction is straightforward. We have two trivialisations of $\delta(\mathcal{G})$, which differ by a line bundle $T_{1} \oslash T_{2} \rightarrow X_{1}$. This gives us $A_{T_{1}}=A_{T_{2}} \otimes \delta\left(T_{1} \oslash T_{2}\right)$ so that $\delta\left(T_{1} \oslash T_{2}\right)$ has a section $s_{1} \otimes s_{2}^{*}$. As $\delta\left(s_{1}\right)=\delta\left(s_{2}\right)=1$ it follows that $\delta\left(s_{1} \otimes s_{2}^{*}\right)=1$.

Proposition 3.12. Let $(T, s)$ be a simplicial extension of $\mathcal{G}$ and $\rho: \mathcal{H} \rightarrow \mathcal{G}$ be a stable isomorphism. Then there is a canonically defined simplicial extension $\rho^{-1}(T, s)$ of $\mathcal{H}$. Moreover $\epsilon\left(\rho^{-1}(T, s)\right)=\epsilon(T, s)$.

3.2. Descent for bundle gerbes. Let $\pi: M \rightarrow N$ be a surjective submersion.

Proposition 3.13. If $\pi: M \rightarrow N$ is a surjective submersion and $\mathcal{G}$ is a bundle gerbe on $N$ then $\pi^{*}(\mathcal{G})$ admits a simplicial extension to $M^{[\bullet+1]}$. 
Proof. The projection $M^{[k]} \rightarrow N=N^{(k)}$ defines a morphism of simplicial manifolds $M^{[\bullet+1]} \rightarrow N^{(\bullet)}$ and it suffices to pull back the simplicial extension defined in Proposition 3.3 .

The converse of Proposition 3.13 is in fact true. Before proving this, we make two definitions as follows:

Definition 3.14. If $M \rightarrow N$ is a surjective submersion and $\mathcal{G}$ a bundle gerbe on $M$ then $(M \rightarrow N)$-descent data for $\mathcal{G}$ is a simplicial extension of $\mathcal{G}$ over $M^{[\bullet+1]}$.

Definition 3.15. If $\pi: M \rightarrow N$ is a surjective submersion and $\mathcal{G}$ a bundle gerbe on $M$ then we say that $\mathcal{G}$ descends to $M$ if there exists a bundle gerbe $\mathcal{H}$ over $N$ with $\pi^{*}(\mathcal{H})$ stably isomorphic to $\mathcal{G}$.

Proposition 3.16. If $\pi: M \rightarrow N$ is a surjective submersion and $\mathcal{G}$ a bundle gerbe on $M$ then $\mathcal{G}$ descends to $N$ if and only if there exists $(M \rightarrow N)$-descent data for $\mathcal{G}$.

Proof. We have established one direction already in Proposition 3.13 Let $\pi: M \rightarrow N$ be a surjective submersion and $\mathcal{G}=(P, Y)$ a bundle gerbe over $M$ with a simplicial extension $(T, s)$ for the simplicial manifold $M^{[\bullet+1]}$. Then let $\bar{Y}=Y \rightarrow N$, regarded as a surjective submersion over $N$. Then there is an equality of simplicial surjective submersions $\bar{Y}^{[\bullet+1]}=\mu^{-1}\left(Y^{\bullet+1}\right)$. The simplicial extension defines a simplicial line bundle over $\mu^{-1}\left(Y^{\bullet+1}\right)$ and hence a simplicial line bundle over $\bar{Y}^{[\bullet+1]}$, i.e. a bundle gerbe. We take the descended bundle gerbe $\mathcal{H}$ to be the one defined by this simplicial line bundle.

We need to check that the pullback of $\mathcal{H}$ is stably isomorphic to $\mathcal{G}$. Consider $\pi^{-1}(\bar{Y}) \rightarrow$ $M$. This contains $Y$ so we have a morphism of simplicial manifolds

$$
Y^{[\bullet+1]} \rightarrow \pi^{-1}\left(\bar{Y}^{[\bullet+1]}\right) \rightarrow \bar{Y}^{[\bullet+1]}=\mu^{-1}\left(Y^{\bullet+1}\right) .
$$

Notice that this composition maps $Y^{[\bullet+1]}$ to the subset $Y^{[\bullet+1]} \subset \mu^{-1}\left(Y^{\bullet+1}\right)$ and is the identity. It follows that if we start with the simplicial extension $(T, s)$ as a simplicial line bundle on $\mu^{-1}\left(Y^{\bullet+1}\right)$, we descend by regarding it as a simplicial line bundle on $\bar{Y}^{[\bullet+1]}$ and we pullback to $\pi^{-1}\left(\bar{Y}^{[\bullet+1]}\right)$ and restrict to $Y^{[\bullet+1]}$, that is the same as just restricting $(T, s)$ to $Y^{[\bullet+1]}$, which by Proposition 3.6 we know to be $(P, Y)$. Hence $\pi^{-1}(\mathcal{H})$ is stably isomorphic to $\mathcal{G}$.

We are interested in several particular cases of simplicial extensions, arising from actions of Lie groups and actions of 2-groups. We devote the rest of this paper to the study of these.

\section{Equivariant BUNDLE GERBES}

4.1. Strong and weak group actions on bundle gerbes. Recall that if $M$ is a manifold on which a Lie group $G$ acts smoothly on the right we have the simplicial manifold $E G(M)$. from Example 2.4. We define:

Definition 4.1. If $G$ acts smoothly on $M$ and $\mathcal{G}$ is a bundle gerbe on $M$ a weak action of $G$ on $\mathcal{G}$ is a simplicial extension for $\mathcal{G}$ over $E G(M)$.

Notice that the simplicial extension class of a weak action lives in $H^{3}(E G(M)$. $\mathbb{R})=$ $H_{G}^{3}(M, \mathbb{R})$, the equivariant de Rham cohomology of $M$.

Definition 4.2 (c.f. 222, 27, 28). Let $\mathcal{G}=(P, Y)$ be a bundle gerbe over $M$. A strong action of $G$ on $\mathcal{G}$ is a smooth action of $G$ on $Y$ covering the action on $M$ and a smooth action of $G$ on $P \rightarrow Y^{[2]}$ by bundle morphisms covering the induced action on $Y^{[2]}$ and commuting with the bundle gerbe product. We say that $\mathcal{G}$ is a strongly equivariant bundle gerbe. 
Proposition 4.3. A strong action of $G$ on $\mathcal{G}$ induces a weak action.

Proof. We construct a simplicial extension $(E G(Y)$. $T, s)$ of $\mathcal{G}=(P, Y)$ over $E G(M)$. First we need a trivialisation of $(\delta(P), Y \times G)$, which has fibre over $\left(y_{1}, y_{2}, g\right) \in Y^{[2]} \times$ $G=(Y \times G) \times_{M \times G}(Y \times G)$ given by $P_{\left(y_{1}, y_{2}\right)}^{*} \otimes P_{\left(y_{1} g, y_{2} g\right)}$. Hence, $(\delta(P), Y \times G)$ is strongly trivial via the isomorphism $P_{\left(y_{1}, y_{2}\right)}^{*} \otimes P_{\left(y_{1} g, y_{2} g\right)} \stackrel{1 \otimes g^{-1}}{\longrightarrow} P_{\left(y_{1}, y_{2}\right)}^{*} \otimes P_{\left(y_{1}, y_{2}\right)} \simeq U(1)$. We therefore take $T$ to be the trivial bundle $(Y \times G) \times U(1)$. Thus $\delta(T)$ is trivial and descends to the trivial bundle with its canonical section $s$, and so $\delta(s)=1$.

4.2. Descent for equivariant bundle gerbes. If $\pi: M \rightarrow N$ is a principal $G$-bundle it is straightforward to show that if a bundle gerbe $\mathcal{G}=(P, Y)$ on $M$ admits a strong action of $G$ then it descends to a quotient bundle gerbe $(P / G, Y / G)$ on $N$. We now show that even for a weak action of $G$ bundle gerbes descend.

Proposition 4.4. Let $M \rightarrow N$ be a principal $G$-bundle. If $\mathcal{G}$ is a bundle gerbe on $M$ acted on weakly by $G$ then $\mathcal{G}$ descends to a bundle gerbe on $N$, which is given explicitly by Proposition 3.16

Proof. The proof is straightforward and only requires us to show that a weak $G$ action on $\mathcal{G}$ is the same as $(M \rightarrow N)$-descent data for $\mathcal{G}$. Equivalently, we need to show that the simplicial manifolds $E G(M)$, and $M^{[\bullet+1]}$ are isomorphic, which is true by Lemma 2.1

A similar result is proved in 21] on the level of cohomology using the definition of equivariance from 7 .

In the case of a strong action we now have two ways to descend the bundle gerbe. These are related by

Proposition 4.5. Let $M \rightarrow N$ be a principal $G$-bundle and $\mathcal{G}=(P, Y)$ a bundle gerbe on $M$ acted on strongly by $G$. The quotient of $\mathcal{G}$ by the strong $G$ action and the descent of $\mathcal{G}$ by the induced weak $G$ action are stably isomorphic.

Proof. We defined the descent of the bundle gerbe $(P, Y)$ in Proposition 3.16 given a weak action of $G$ in the form of a simplicial extension $\left(\mu^{-1}\left(Y^{\bullet+1}\right), T, s\right)$ of $(P, Y)$ over $M^{[\bullet+1]}$. This was given by the observation that $\mu^{-1}\left(Y^{\bullet+1}\right)=\bar{Y}^{[\bullet+1]}$ (for $\bar{Y} \rightarrow M / G$ the submersion given by the composition $Y \rightarrow M \rightarrow M / G)$ and then pulling back the simplicial extension to a simplicial line bundle $[T, s]$ over $\bar{Y}^{[\bullet+1]}$. We also have a quotient bundle gerbe $(P / G, Y / G)$ over $M / G$. We define a map of surjective submersions $\rho: \bar{Y} \rightarrow Y / G$ over $N=M / G$ and show that $\rho^{-1}(P / G) \simeq T$ and that this map commutes with the bundle gerbe multiplication on $T \rightarrow \bar{Y}^{[2]}$ and $P / G \rightarrow(Y / G)^{[2]}$.

Notice, however, that the weak action on $(P, Y)$ induced by the strong action of $G$, given in Proposition 4.3 is a simplicial extension $(E G(Y), Y \times U(1), 1)$. Proposition 3.5 tells us that this is the pullback of a simplicial extension $\left(\mu^{-1}\left(Y^{\bullet+1}\right), T, s\right)$. Therefore, we need the simplicial line bundle coming from this simplicial extension. The trivialisation $T$ is given by Proposition A.1 as follows. We have the map $E G(Y)_{1}=Y \times G \rightarrow$ $\mu^{-1}\left(Y^{\bullet+1}\right)=\bar{Y}^{[2]} ;(y, g) \mapsto(y, y g)$. Then for $\left(y_{0}, y_{1}\right),\left(y_{0}^{\prime} \cdot y_{1}^{\prime}\right) \in \bar{Y}^{[2]} \times_{M \times G} \bar{Y}^{[2]}$ we have $\delta(P)_{\left(y_{0}, y_{1}\right),\left(y_{0}^{\prime}, y_{1}^{\prime}\right)}=P_{\left(y_{0}, y_{0}^{\prime}\right)}^{*} \otimes P_{\left(y_{1}, y_{1}^{\prime}\right)}$. Note that $\pi\left(y_{1}\right)=\pi\left(y_{0}\right) g$ for some $g \in G$ and $\pi: Y \rightarrow M$, and similarly for $y_{0}^{\prime}, y_{1}^{\prime}$, and that $\pi\left(y_{i}\right)=\pi\left(y_{i}^{\prime}\right)$. Then Proposition A.1 gives

$$
\begin{aligned}
T_{\left(y_{0}, y_{1}\right)} & =U(1) \otimes \delta(P)_{(y, y g)\left(y_{0}, y_{1}\right)} \\
& =U(1) \otimes P_{\left(y, y_{0}\right)}^{*} \otimes P_{\left(y g, y_{1}\right)} \\
& =U(1) \otimes P_{\left(y, y_{0}\right)}^{*} \otimes P_{\left(y, y_{1} g^{-1}\right)} \\
& =U(1) \otimes P_{\left(y_{0}, y_{1} g^{-1}\right)} \\
& =P_{\left(y_{0}, y_{1} g^{-1}\right)}
\end{aligned}
$$


where again $g$ is such that $\pi\left(y_{1}\right)=\pi\left(y_{0}\right) g$. To construct the descended bundle gerbe we need the section of $\delta(T) \rightarrow \bar{Y}^{[3]}$. For $\left(y_{0}, y_{1}, y_{2}\right) \in \bar{Y}^{[3]}$ and $\pi\left(y_{i}\right)=\pi\left(y_{j}\right) g_{i j}$ we have

$$
\delta(T)_{\left(y_{0}, y_{1}, y_{2}\right)}=P_{\left(y_{1}, y_{2} g_{12}^{-1}\right)} \otimes P_{\left(y_{0}, y_{2} g_{02}^{-1}\right)}^{*} \otimes P_{\left(y_{0}, y_{1} g_{01}^{-1}\right)},
$$

and a section is given by $s\left(y_{0}, y_{1}, y_{2}\right)=p_{12} \otimes\left(p_{12} g_{01} \cdot p_{01}\right)^{*} \otimes p_{01}$, where $p_{01} \in P_{\left(y_{0}, y_{1}\right)}, p_{12} \in$ $P_{\left(y_{1}, y_{2}\right)}$ and $\cdot$ denotes the bundle gerbe multiplication in $P$.

We can now define $\rho: \bar{Y} \rightarrow Y / G$ by $\rho(y)=y G \in Y / G$ where the latter denotes the orbit of $y$ under $G$. Then we have a map $T_{\left(y_{0}, y_{1}\right)}=P_{\left(y_{0}, y_{1} g^{-1}\right)} \rightarrow(P / G)_{\left(y_{0} G, y_{1} G\right)}$ because the $G$ orbit of $\left(y_{0}, y_{1} g^{-1}\right)$ is the pair of $G$ orbits $\left(y_{0} G, y_{1} g^{-1} G\right)=\left(y_{0} G, y_{1} G\right)$. Hence we have described a bundle map $T \rightarrow P / G$ covering the induced map $\rho: \bar{Y}^{[2]} \rightarrow(P / G)^{[2]}$.

We need to prove that this map preserves the bundle gerbe product. The multiplication in $(P / G, Y / G)$ is given by the section $\sigma\left(y_{0} G, y_{1} G, y_{2} G\right)=p_{12} \otimes\left(p_{12} \cdot p_{01} G\right) \otimes p_{01}$ and it is easy to see that $\rho$ maps the section $s$ to $\sigma$ because the $G$ action on $P$ commutes with the bundle gerbe multiplication. It follows that the bundle gerbe product is preserved.

4.3. The class of a strongly equivariant bundle gerbe. Assume that $G$ acts strongly on the bundle gerbe $\mathcal{G}=(P, Y)$ over $M$. Choose a bundle gerbe connection $\nabla$ for $P$ and a curving $f$. Let $\omega_{(3,0)} \in \Omega^{3}(M)$ be the three-curving. We show how to write down an equivariant three-class for $\mathcal{G}$.

Over $Y^{[2]} \times G$ there are two bundles $d_{0}^{-1}(P)$ and $d_{1}^{-1}(P)$ corresponding to the bundle gerbes $d_{0}^{-1}(\mathcal{G})$ and $d_{1}^{-1}(\mathcal{G})$ over $M \times G$. Let $\phi: d_{0}^{-1}(P) \rightarrow d_{1}^{-1}(P)$ be the action of right multiplication by $g^{-1}$. On $d_{0}^{-1}(P)$ there are two connections: $d_{0}^{-1}(\nabla)$ and $\phi^{-1} d_{1}^{-1}(\nabla) \phi$. They are both bundle gerbe connections so we must have

$$
d_{0}^{-1}(\nabla)-\phi^{-1} d_{1}^{-1}(\nabla) \phi=\delta_{Y}(\beta),
$$

for $\beta \in \Omega^{1}(Y \times G)$. Similarly we have curvings $d_{0}^{*}(f)$ and $d_{1}^{*}(f)$ and

$$
\delta_{Y}\left(d_{0}^{*}(f)-d_{1}^{*}(f)-d \beta\right)=0,
$$

or

$$
d_{0}^{*}(f)-d_{1}^{*}(f)-d \beta=\pi^{*}\left(\omega_{(2,1)}\right),
$$

for $\omega_{(2,1)} \in \Omega^{1}(M \times G)$. Moreover

$$
\begin{aligned}
\pi^{*}\left(d \omega_{(2,1)}\right) & =d_{0}^{*}(f)-d_{1}^{*}(f) \\
& =d_{0}^{*}(d f)-d_{1}^{*}(d f) \\
& =d_{0}^{*}\left(\pi^{*}\left(\omega_{(3,0)}\right)\right)-d_{1}^{*}\left(\pi^{*}\left(\omega_{(3,0)}\right)\right)
\end{aligned}
$$

so that

$$
-d \omega_{(2,1)}+\delta\left(\omega_{(3,0)}\right)=0 .
$$

Applying $d_{0}^{-1}, d_{1}^{-1}$ and $d_{2}^{-1}$ to 4.1 we obtain

$$
\begin{aligned}
& \mu_{2}^{-1}(\nabla)-\phi_{0}^{-1} \mu_{1}^{-1}(\nabla) \phi_{0}=\delta_{Y}\left(d_{0}^{*}(\beta)\right) \\
& \mu_{2}^{-1}(\nabla)-\phi_{1}^{-1} \mu_{0}^{-1}(\nabla) \phi_{1}=\delta_{Y}\left(d_{1}^{*}(\beta)\right) \\
& \mu_{1}^{-1}(\nabla)-\phi_{2}^{-1} \mu_{0}^{-1}(\nabla) \phi_{2}=\delta_{Y}\left(d_{2}^{*}(\beta)\right)
\end{aligned}
$$

where here $\mu_{0}\left(m, g_{1}, g_{2}\right)=m, \mu_{1}\left(m, g_{1}, g_{2}\right)=m g_{1}$ and $\mu_{2}\left(m, g_{1}, g_{2}\right)=m g_{1} g_{2}$ and $\phi_{i}=$ $\phi \circ d_{i}$. We have $\phi_{1}=\phi_{2} \phi_{0}$ so conjugating line (4.3) by $\phi_{0}^{-1}$ we obtain

$$
\phi_{0}^{-1} \mu_{1}^{-1}(\nabla) \phi_{0}-\phi_{1}^{-1} \mu_{0}^{-1}(\nabla) \phi_{1}=\delta_{Y}\left(d_{2}^{*}(\beta)\right),
$$

and an alternating sum gives us $\delta_{Y}(\delta(\beta))=0$. Hence

$$
\delta(\beta)=\pi^{*}\left(\omega_{(1,2)}\right),
$$


for some $\omega_{(1,2)} \in \Omega^{1}\left(M \times G^{2}\right)$. It then follows that

$$
\pi^{*}\left(\delta\left(\omega_{(2,1)}\right)\right)=-d \delta(\beta)=-\pi^{*}\left(d \omega_{(1,2)}\right),
$$

or

$$
d \omega_{(1,2)}+\delta\left(\omega_{(2,1)}\right)=0 .
$$

Notice also that $0=\delta^{2}(\beta)=-\pi^{*}\left(\delta\left(\omega_{(1,2)}\right)\right)$ so that $\delta\left(\omega_{(1,2)}\right)=0=d 0$. Thus we have defined a cocycle

$$
\omega=\left(0, \omega_{(1,2)}, \omega_{(2,1)}, \omega_{(3,0)}\right) \in \mathcal{A}^{3}(E G(M) \bullet) .
$$

Consider what happens if we vary the choices involved. We could replace $\beta$ by $\beta+$ $\pi^{*}\left(\rho_{(1,1)}\right)$, changing $\omega_{(2,1)}$ by adding $-d \rho_{(1,1)}$ and $\omega_{(1,2)}$ by adding $\delta\left(\rho_{(1,1)}\right)$, which leaves the class of $\omega$ unchanged. Also we could replace the curving $f$ by adding $\pi^{*}\left(\rho_{(2,0)}\right)$ to it and changing $\omega_{(3,0)}$ by addition of $d \rho_{(2,0)}$, and $\omega_{(2,1)}$ by addition of $\delta\left(\rho_{(2,0)}\right)$, which again leaves the class of $\omega$ unchanged. Finally, we can change $(\nabla, f)$ to $(\nabla+\delta(\alpha), f+d \alpha)$ for $\alpha \in \Omega^{1}(Y)$, which changes $\beta$ to $\beta+\delta(\alpha)$. The left hand side of equation 4.2 then changes by the addition of $\delta(d \alpha)-d \delta(\alpha)=0$, leaving $\omega$ unchanged. We conclude that the class of $\omega$ depends only on the strong group action and the bundle gerbe.

Definition 4.6. We call the class just defined the strongly equivariant class of the strongly equivariant bundle gerbe $\mathcal{G}$ and denote it by $\epsilon_{s}(\mathcal{G})$.

In 28] Meinrenken defines the class of a strongly equivariant bundle gerbe using the Cartan model of equivariant cohomology. See also related work of Stienon [38.

With these observations we can prove the following result:

Proposition 4.7. The equivariant class of a strongly equivariant gerbe is equal to the simplicial extension class of the corresponding simplicial extension.

Proof. In Proposition 3.7 we constructed the class $\eta=\left(0, \eta_{(1,2)}, \eta_{(2,1)}, \eta_{(3,0)}\right)$, which we compare to the class $\omega=\left(0, \omega_{(1,2)}, \omega_{(2,1)}, \omega_{(3,0)}\right)$ above.

Firstly, it is clear that $\omega_{(3,0)}=\eta_{(3,0)}$.

Recall from Proposition 4.3 that the simplicial extension corresponding to the strong action of $G$ on $\mathcal{G}$ is given by $(E G(Y) \bullet, T, s)$, where $T$ (and hence $A_{T}$ ) is the trivial bundle and $s$ is the canonical section of $A_{T}$. Equation (4.1) tells us we can choose the trivialising connection on $T$ to be $\beta$ and then equation $(4.2)$ is the same as equation 3.2 and hence $\eta_{(2,1)}=\omega_{(2,1)}$.

Finally, the induced connection on $\delta(T)$ is given by $\delta(\beta)$ and hence comparing equation 4.4 with equation (3.3) implies that $\eta_{(1,2)}=\omega_{(1,2)}$.

\section{The BASIC BUndLe Gerbe}

We review the constructions in 34 and situate them in the equivariant setting. We first recall from 34 the basic bundle gerbe on $G=U(n)$ and the canonical connection and curving on it constructed using the holomorphic functional calculus.

Write $Z=S^{1} \backslash\{1\}$. Define $Y \subset Z \times G$ to be the set of pairs $(z, g)$, where $z$ is not an eigenvalue of $g$. We equip $Z$ with an ordering via the identification of $Z$ with the open interval $(0,2 \pi)$ by $\phi \mapsto \exp (i \phi)$. Let $\pi: Y \rightarrow G$ denote the canonical map. We note that elements of $Y^{[2]}$ can be identified with triples $\left(z_{1}, z_{2}, g\right)$ where $\left(z_{1}, g\right),\left(z_{2}, g\right) \in Y$. In such a case if $z \in Z$ we say that it is between $z_{1}$ and $z_{2}$ if it is in the component of $S^{1} \backslash\left\{z_{1}, z_{2}\right\}$ not containing $\{1\}$.

As described in [34, there is a natural line bundle $L$ on $Y^{[2]}$ together with a bundle gerbe product on $L$, giving $(L, Y)$ the structure of a bundle gerbe on $G$. To describe this note first that there is a decomposition of $Y^{[2]}$ as a union of three disjoint open sets defined by:

$$
\begin{aligned}
& Y_{+}^{[2]}=\left\{\left(z_{1}, z_{2}, g\right) \mid z_{1}<z_{2} \text { and there is some eigenvalue of } g \text { between } z_{1} \text { and } z_{2}\right\} \\
& Y_{-}^{[2]}=\left\{\left(z_{1}, z_{2}, g\right) \mid z_{1}>z_{2} \text { and there is some eigenvalue of } g \text { between } z_{1} \text { and } z_{2}\right\}
\end{aligned}
$$


and

$Y_{0}^{[2]}=\left\{\left(z_{1}, z_{2}, g\right) \mid\right.$ there is no eigenvalue of $g$ between $z_{1}$ and $\left.z_{2}\right\}$.

If $\left(z_{1}, z_{2}, g\right) \in Y_{+}^{[2]}$ we define

$$
E_{\left(z_{1}, z_{2}, g\right)}=\bigoplus_{z_{1}<\lambda<z_{2}} E_{\lambda}(g)
$$

where $E_{\lambda}(g)$ denotes the $\lambda$-eigenspace of $g$ and we write $z_{1}<\lambda<z_{2}$ to indicate that $\lambda$ is between $z_{1}$ and $z_{2}$. It is shown in 34 that $E \rightarrow Y_{+}^{[2]}$ is a smooth, locally trivial vector bundle. Recall also from [34] that the orthogonal projection $P: Y_{+}^{[2]} \rightarrow M_{n}(\mathbb{C})$ onto $E$ is given by the contour integral formula

$$
P\left(z_{1}, z_{2}, g\right)=\frac{1}{2 \pi i} \oint_{C_{\left(z_{1}, z_{2}, g\right)}}(\xi 1-g)^{-1} d \xi
$$

where $C_{\left(z_{1}, z_{2}, g\right)}$ is an anti-clockwise curve enclosing all of the eigenvalues of $g$ between $z_{1}$ and $z_{2}$.

The line bundle $L \rightarrow Y^{[2]}$ is defined as follows. If $\left(z_{1}, z_{2}, g\right) \in Y_{+}^{[2]}$ we set

$$
L_{\left(z_{1}, z_{2}, g\right)}=\bigwedge^{\text {top }} E_{\left(z_{1}, z_{2}, g\right)} .
$$

If $\left(z_{1}, z_{2}, g\right) \in Y_{-}^{[2]}$ we set $L_{\left(z_{1}, z_{2}, g\right)}=L_{\left(z_{2}, z_{1}, g\right)}^{*}$. If $(z, z, g) \in Y_{0}^{[2]}$ we set $L_{(z, z, g)}=\mathbb{C}$. It is proven in [34, Proposition 3.1] that $L \rightarrow Y^{[2]}$ is a smooth, locally trivial, hermitian line bundle. Furthermore it is shown in 34] that there is a natural bundle gerbe product on $L$, equipping $(L, Y)$ with the structure of a bundle gerbe. The resulting bundle gerbe $\mathcal{B}_{n}=(L, Y)$ is a model for the basic bundle gerbe on $G=U(n)$.

Observe that $G$ acts smoothly on $Y$ from the right, covering the adjoint action of $G$ on itself. More precisely, we define $Y \times G \rightarrow Y$ by $((z, g), h) \mapsto\left(z, h^{-1} g h\right)$; note that the projection map $\pi: Y \rightarrow G$ is equivariant. We have the following lemma.

Lemma 5.1. The basic bundle gerbe $\mathcal{B}_{n}=(L, Y)$ on $G=U(n)$ is a strongly equivariant bundle gerbe for the adjoint action of $G$ on itself.

Proof. We need to show that the induced action $Y^{[2]} \times G \rightarrow Y^{[2]}$ lifts to an action of $G$ on $L$. It is sufficient to prove that the action of $G$ on $Y_{+}^{[2]}$ lifts to an action of $G$ on $E$; this follows from the fact that the left action of $G$ on $\mathbb{C}^{n}$ is smooth and the fact that if $v$ is an eigenvector of $g$ with eigenvalue $\lambda$, then $v h$ is an eigenvector of $h^{-1} g h$ with eigenvalue $\lambda$.

The map $P: Y_{+}^{[2]} \rightarrow M_{n}(\mathbb{C})$ extends in an obvious way to a smooth map $P: Y^{[2]} \rightarrow$ $M_{n}(\mathbb{C})$. Observe that $P$ satisfies

$$
d_{1}^{*} P=\operatorname{Ad}_{p_{2}} d_{0}^{*} P
$$

on $Y^{[2]} \times G$, where $p_{2}: Y^{[2]} \times G \rightarrow G$ is the map $p_{2}\left(\left(z_{1}, z_{2}, g\right), h\right)=h$.

Recall from 34 that there is a canonical bundle gerbe connection $\nabla$ and curving $f$ on $(L, Y)$ whose 3 -curvature is equal to the basic 3 -form

$$
\nu=-\frac{1}{24 \pi^{2}} \operatorname{tr}\left(g^{-1} d g\right)^{3} .
$$

We briefly review the construction of $\nabla$ and $f$ as they will be needed in the sequel. The orthogonal projection $P: Y_{+}^{[2]} \rightarrow M_{n}(\mathbb{C})$ induces a connection $\nabla_{E}$ on $E$ by projecting the trivial connection $d$ on $Y_{+}^{[2]} \times \mathbb{C}^{n}$ to $E$. The connection $\nabla_{E}$ then induces a connection $\nabla$ on the restriction of $L$ to $Y_{+}^{[2]}$, over $Y_{-}^{[2]}$ we equip $L$ with the dual connection and over $Y_{0}^{[2]}$ we take the flat connection. It is proven in 34 that this connection $\nabla$ on $L$ is a 
bundle gerbe connection and that moreover a curving $f$ for $\nabla$ is given by the 2-form on $Y$ defined by

$$
f(g, z)=\frac{1}{8 \pi^{2}} \oint_{C_{(g, z)}} \log _{z} \xi \operatorname{tr}\left((\xi 1-g)^{-1} d g(\xi 1-g)^{-2} d g\right) d \xi
$$

where $C_{(g, z)}$ is an anti-clockwise contour in $\mathbb{C} \backslash R_{z}$ enclosing the eigenvalues of $g$, and where $R_{z}$ denotes the closed ray from the origin in $\mathbb{C}$ through $z$. Here $\log _{z}: \mathbb{C} \backslash R_{z} \rightarrow \mathbb{C}$ is the branch of the logarithm defined by making the cut along $R_{z}$ and defining $\log _{z}(1)=0$.

The connection $\nabla$ on $L$ is not equivariant however, for the action of $G$ on $L$ described in Lemma 5.1 above. We investigate the failure of $\nabla$ to be equivariant more closely. We have an isomorphism of line bundles $\phi: d_{0}^{*} L \rightarrow d_{1}^{*} L$ over $Y^{[2]} \times G$; if $s$ is a section of $d_{0}^{*} L$ over $Y^{[2]} \times G$ then $\phi(s)$ is the section of $d_{1}^{*} L$ over $Y^{[2]} \times G$ defined by

$$
\phi(s)=s \cdot p_{2},
$$

where $\left(s \cdot p_{2}\right)\left(\left(z_{1}, z_{2}, g\right), h\right)=s\left(z_{1}, z_{2}, g\right) \cdot h$. We then have

$$
\begin{aligned}
\phi^{-1} d_{0}^{*} \nabla(\phi(s)) & =\phi^{-1} \operatorname{det}\left(d_{0}^{*} P\left(d\left(s \cdot p_{2}\right)\right)\right) \\
& =\phi^{-1} \operatorname{det}\left(d_{0}^{*} P\left(d s \cdot p_{2}+s \cdot d p_{2}\right)\right) \\
& =\phi^{-1} \operatorname{det}\left(\operatorname{Ad}_{p_{2}} d_{0}^{*} P\left(d s \cdot p_{2}+s \cdot d p_{2}\right)\right) \\
& =\operatorname{tr}\left(p_{2}^{*} \theta d_{1}^{*} P\right) \cdot s+d_{1}^{*} \nabla s,
\end{aligned}
$$

where we have used (5.2) and where we have written $\theta$ for the right Maurer-Cartan 1-form on $G$. Using (5.1) we may express the 1-form $\alpha=\operatorname{tr}\left(p_{2}^{*} \theta d_{1}^{*} P\right)$ as a contour integral:

$$
\alpha\left(z_{1}, z_{2}, g, h\right)=\frac{1}{2 \pi i} \oint_{C_{\left(g, z_{1}, z_{2}\right)}} \operatorname{tr}\left(\theta(h)(\xi 1-g)^{-1}\right) d \xi,
$$

where, as in 5.1) above, $C_{\left(g, z_{1}, z_{2}\right)}$ denotes a contour enclosing the eigenvalues of $g$ between $z_{1}$ and $z_{2}$, oriented counter-clockwise.

Since $d_{0}^{*} \nabla$ and $d_{1}^{*} \nabla$ are bundle gerbe connections, it follows that $\delta(\alpha)=0$ and hence $\alpha=\delta(\beta)$ for some 1-form $\beta$ on $Y \times G$. Using an identical argument to that used in the proof of part (a) of Theorem 5.1 in [34, we obtain the following expression for $\beta$ :

$$
\beta(z, g, h)=-\frac{1}{4 \pi^{2}} \oint_{C_{(g, z)}} \log _{z} \xi \operatorname{tr}\left(\theta(h)(\xi 1-g)^{-1}\right) d \xi
$$

where $\log _{z}$ and $C_{(g, z)}$ are respectively the branch of the logarithm and the contour described above.

The main result of this section is the following theorem.

Theorem 5.2. Let $G=U(n)$. Then the strongly equivariant bundle gerbe $(L, Y)$ has an equivariant bundle gerbe connection and curving given by $(\nabla, f, \beta)$, where $\nabla$ is the bundle gerbe connection on $L$ described above, $f$ is the curving 2-form on $Y$ given by

$$
f(z, g)=\frac{1}{8 \pi^{2}} \oint_{C_{(g, z)}} \log _{z} \xi \operatorname{tr}\left((\xi 1-g)^{-1} d g(\xi 1-g)^{-2} d g\right) d \xi
$$

and $\beta$ is the 1 -form on $Y \times G$ given by

$$
\beta(z, g, h)=-\frac{1}{4 \pi^{2}} \oint_{C_{(g, z)}} \log _{z} \xi \operatorname{tr}\left(\theta(h)(\xi 1-g)^{-1}\right) d \xi
$$

where the contour $C_{(g, z)}$ and the branch of the logarithm are described as above. Furthermore, the equivariant 3 -curvature of this connection and curving is the cocycle $(\nu, \omega, 0,0) \in$ $\mathcal{A}^{3}(E G(G)$ •) where

$$
\begin{aligned}
& \nu=-\frac{1}{24 \pi^{2}} \operatorname{tr}\left(g^{-1} d g\right)^{3} \in \Omega^{3}(G) \\
& \omega=\frac{i}{4 \pi}\left(\operatorname{tr}\left(\hat{\theta}_{h} \theta_{h}\right)+\operatorname{tr}\left(\theta \theta_{h}\right)+\operatorname{tr}\left(\theta \hat{\theta}_{h}\right)\right) \in \Omega^{2}\left(G^{2}\right)
\end{aligned}
$$


where we have defined $\theta=g^{-1} d g, \theta_{h}=d h h^{-1}$ and $\hat{\theta}_{h}=g^{-1} \theta_{h} g$. Hence the strongly equivariant class of $\mathcal{B}_{n}$ is $\epsilon_{s}\left(\mathcal{B}_{n}\right)=[\nu, \omega, 0,0]$.

Proof. We need to show that the following equations hold:

$$
\begin{aligned}
& \delta(\nabla)=\delta(\beta) \\
& \delta(f)-d \beta=\pi^{*} \omega \\
& \delta(\beta)=0 .
\end{aligned}
$$

We have established the first equation in the paragraphs preceding the statement of the theorem. We show that the third equation is satisfied, i.e. that $\delta(\beta)=0$. We have $d_{0}^{*} \beta((z, g), h, k)=\beta\left(\left(z, h^{-1} g h\right), k\right), d_{1}^{*} \beta((z, g), h, k)=\beta((z, g), h k)$ and $d_{2}^{*} \beta((z, g), h, k)=$ $\beta((z, g), h)$. Therefore we have that $\delta(\beta)(z, g, h, k)$ is equal to

$$
\frac{1}{4 \pi^{2}} \oint_{C_{(g, z)}} \log _{z} \xi \operatorname{tr}\left[\theta(k) h^{-1}(\xi 1-g)^{-1} h-\theta(k h)(\xi 1-g)^{-1}+\theta(h)(\xi 1-g)^{-1}\right] d \xi,
$$

which is easily seen to equal 0 using $\theta(h k)=h \theta(k) h^{-1}+\theta(h)$. The proof that the second equation is satisfied is long and technical and we have therefore relegated it to Appendix B

As an illustration of this theorem we consider the case where $G=U(1)$ in detail. In this case the bundle gerbe on $G$ is necessarily trivial. However, the equivariant bundle gerbe on $G$ is non-trivial. The theorem above shows that its equivariant 3 -curvature is given by $\left(0, \omega_{(2,1)}\right)$, where $\omega_{(2,1)}$ is the closed 2 -form on $U(1) \times U(1)$ given by

$$
\omega_{(2,1)}\left(\phi_{1}, \phi_{2}\right)=\frac{1}{4 \pi^{2}} d \phi_{1} \wedge d \phi_{2}
$$

where we have defined $g_{1}=\exp \left(i \phi_{1}\right)$ and $g_{2}=\exp \left(i \phi_{2}\right)$. An easy calculation shows that the class in $H^{2}(U(1) \times U(1), \mathbb{R})$ represented by $\omega_{(2,1)}$ is non-zero. It follows that the class in $H_{U(1)}^{3}(U(1), \mathbb{R})$ represented by $\left(0, \omega_{(2,1)}\right)$ is non-zero.

Note that there also is a non-trivial multiplicative bundle gerbe on $U(1)$, with trivial underlying bundle gerbe, using the line bundle on $U(1) \times U(1)$ with curvature $\omega_{(2,1)}$ [17] .

\section{String Structures And Simplicial EXtensions}

Waldorf 41 has described string structures on a principal $G$-bundle $P \rightarrow X$ as trivialisations of a certain bundle 2-gerbe, called the Chern-Simons bundle 2-gerbe of $P$. In this section we show that such a trivialisation gives rise to a simplicial extension of a bundle gerbe. Unlike the examples so far, this is an example of a simplicial extension over a simplicial manifold that is not the nerve of a Lie groupoid.

6.1. Crossed modules. We shall begin by recording some relevant facts about crossed modules, which will be important in what follows. Crossed modules were introduced by Whitehead in the 1940's as a model for homotopy 2-types. We first recall the definition.

Definition 6.1. A crossed module $\mathcal{K}$ is a pair of groups $\hat{K}$ and $L$ together with a homomorphism $\hat{K} \stackrel{t}{\rightarrow} L$ and an action $L \times \hat{K} \stackrel{\alpha}{\rightarrow} \hat{K}$ by group automorphisms satisfying

(1) $t(\alpha(l, k))=\operatorname{Ad}_{l}(t(k))$,

(2) $\alpha\left(t\left(k_{1}\right), k_{2}\right)=\operatorname{Ad}_{k_{1}}\left(k_{2}\right)$,

for $l \in L$ and $k, k_{1}, k_{2} \in \hat{K}$. We shall further assume that $\hat{K} \rightarrow K$ is a locally trivial principal ker $t$-bundle, where here $K:=t(\hat{K})$.

Remark 6.1. Although we do not need this point of view we remark that a crossed module gives rise to a groupoid $\hat{K} \times L \rightrightarrows L$ where both the objects and arrows are groups and the source and target are homomorphisms. Further, there are functors $1 \rightarrow \mathcal{K}$ and $\mathcal{K} \times \mathcal{K} \rightarrow \mathcal{K}$ making certain natural diagrams commute. Such a thing is the same as a group object in the category of groupoids, and is called a strict 2-group. A detailed 
discussion of this would take us too far afield, so we instead refer the interested reader to history, discussion and definitions in [1]

We also want to say what it means for a crossed module to act on a manifold.

Definition 6.2. A strict action of a crossed module $\hat{K} \stackrel{t}{\rightarrow} L$ on a manifold $P$ is an ordinary group action of $L$ on $P$ such that the action restricted to $K<L$ is trivial.

Remark 6.2. Although Definition 6.2 will suffice for our purposes, we remark that there is a definition of a strict action of a strict 2 -group $\mathcal{K}$ on a manifold $P$ given in terms of a functor $\mathcal{K} \times P \rightarrow P$ (where $P$ is considered as a groupoid with no non-identity arrows) making certain diagrams commute. In the case that the 2 -group $\mathcal{K}$ comes from a crossed module $\hat{K} \stackrel{t}{\rightarrow} L$ it is easy to see that this is equivalent to Definition 6.2

Example 6.1. The crossed module in which we are interested is the following [2]: $\hat{K}$ is the central extension of the loop group $\widehat{\Omega G}$, and $L$ is the path group $P G$. The map $\widehat{\Omega G} \stackrel{t}{\rightarrow} P G$ is the composition of the projection to $\Omega G$ with the inclusion $\Omega G \hookrightarrow P G$ (so $K=t(\hat{K})=\Omega G$ and ker $t=U(1))$ and the map $\alpha: P G \times \widehat{\Omega G} \rightarrow \widehat{\Omega G}$ is a lift of the adjoint map Ad: $P G \times \Omega G \rightarrow \Omega G$, which we also denote by Ad. The result of [2] is that this defines a crossed module that gives a 2-group model for the 3-connected cover of $G$, the String group of $G$.

Let $N$ be a manifold with a $G$-action. The crossed module in the previous paragraph acts on $N$ in a natural way via the evaluation map $P G \rightarrow G$.

This crossed module will be important in what follows since the simplicial manifold we consider in Section 6.4 is built from the crossed module action on the total space of a $G$-bundle.

We have the following facts about $\hat{K} \stackrel{t}{\rightarrow} L$ :

(1) Since $\hat{K}$ is a central extension of $K$ it is multiplicative as a principal bundle, that is the following diagram is a pullback

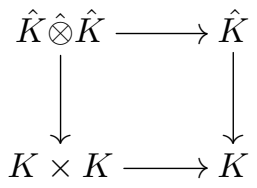

where $\hat{K} \hat{\otimes} \hat{K}$ denotes the external tensor product, $\hat{K}_{1} \otimes \hat{K}_{2}$, where $\hat{K}_{i}$ is the pullback of $\hat{K}$ by the projection onto the $i^{\text {th }}$ factor.

(2) Since $\hat{K} \stackrel{t}{\rightarrow} L$ is a crossed module, the map $\alpha$ lifts the restriction to $K$ of the adjoint map, $\left.\operatorname{Ad}\right|_{K}: L \times K \rightarrow K$, so the following diagram is a pullback

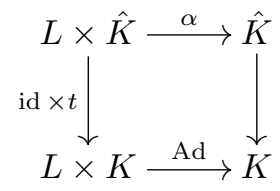

(3) The natural map from the dual bundle $\hat{K}^{*}$ to $\hat{K}$ covers the inverse map on $K$ so that the following diagram is a pullback

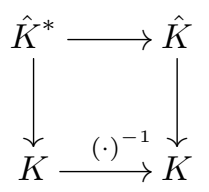

In terms of the fibres of $\hat{K}$ these tell us there are canonical isomorphisms 
(1) $\hat{K}_{k_{1} k_{2}} \simeq \hat{K}_{k_{1}} \otimes \hat{K}_{k_{2}}$,

(2) $\hat{K}_{\mathrm{Ad}_{l}(k)} \simeq \hat{K}_{k}$,

(3) $\hat{K}_{k^{-1}} \simeq \hat{K}_{k}^{*}$

where $l \in L$ and $k \in K$.

We will be concerned with bundles over $L^{n} \times K^{m}$ defined by (products and compositions of) pullbacks of the maps above. We will call such a bundle an xm-bundle. More precisely, we make the following definition.

Definition 6.3. Let $f: L^{n} \times K^{m} \rightarrow K$ be a map given by composition of the following operations:

(1) multiplication in $L$ and $K$;

(2) inversion in $L$ and $K$;

(3) inclusions $K \hookrightarrow L$ and $1 \hookrightarrow K$;

(4) projections $L^{n} \times K^{m} \rightarrow L^{p} \times K^{q}$;

(5) diagonals $L \times K \rightarrow L^{p} \times K^{q}$;

(6) the adjoint action Ad: $L \times K \rightarrow K$.

We call $P \rightarrow L^{n} \times K^{m}$ an $x m$-bundle if $P \simeq f^{-1}(\hat{K})$ for some $f$ as above. Additionally, we define an $x m$-morphism between $\mathrm{xm}$-bundles on the same base to be a map of bundles built from compositions and products of the three structural maps of the crossed module (6.1) - 6.3 above. An xm-morphisms is clearly an isomorphism, since maps of principal bundles are so.

We have the following result, which we will use repeatedly.

Lemma 6.4. There exists at most one xm-morphism between any two xm-bundles.

Proof. To prove this we first make the following observation: Suppose $P$ is an $\mathrm{xm}$-bundle that is the pullback of a map $f: L^{n} \times K^{m} \rightarrow K$. We can factor $f$ through a product of $K$ 's by leaving all the multiplication maps in $f$ until last; that is, we can write $f$ as a $k$-tuple $\left(f_{0}, \ldots, f_{k}\right): L^{n} \times K^{m} \rightarrow K^{k}$, composed with the map $K^{k} \stackrel{m}{\rightarrow} K$ given by multiplication. The maps $f_{1}, \ldots, f_{k}$ do not contain among them any multiplication maps in $K$. Moreover, since Ad is a homomorphism we can further factorise the map $\left(f_{0}, \ldots, f_{k}\right)$ through $L^{\ell} \times K^{k}$ by leaving all the maps involving Ad until last; so $\left(f_{0}, \ldots, f_{k}\right)$ is given by a composition $L^{n} \times K^{m} \stackrel{g}{\rightarrow} L^{\ell} \times K^{k} \stackrel{a}{\rightarrow} K^{k}$, where we have denoted by $a$ the map involving all adjoints and by $g$ the map comprised of all other structure. As above, denote by $\hat{K}^{\otimes} \hat{\otimes}$ the external tensor product of $\hat{K}$ with itself over $K^{k}$. Then the pullback diagram 6.2 implies that the following diagram is a pullback

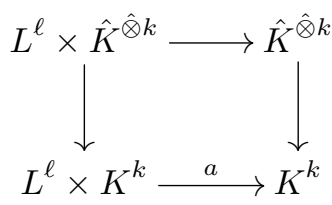

On the other hand, if we consider the map $L^{\ell} \times K^{k} \stackrel{\mathrm{pr}}{\longrightarrow} K^{k} \stackrel{m}{\longrightarrow} K$ given by projection and then multiplication, we see that the following diagram is also a pullback

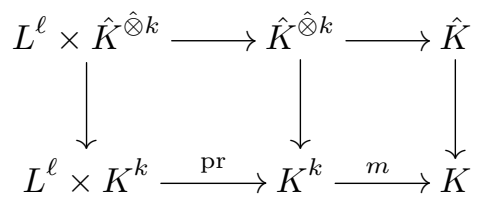


This gives the commutative diagram

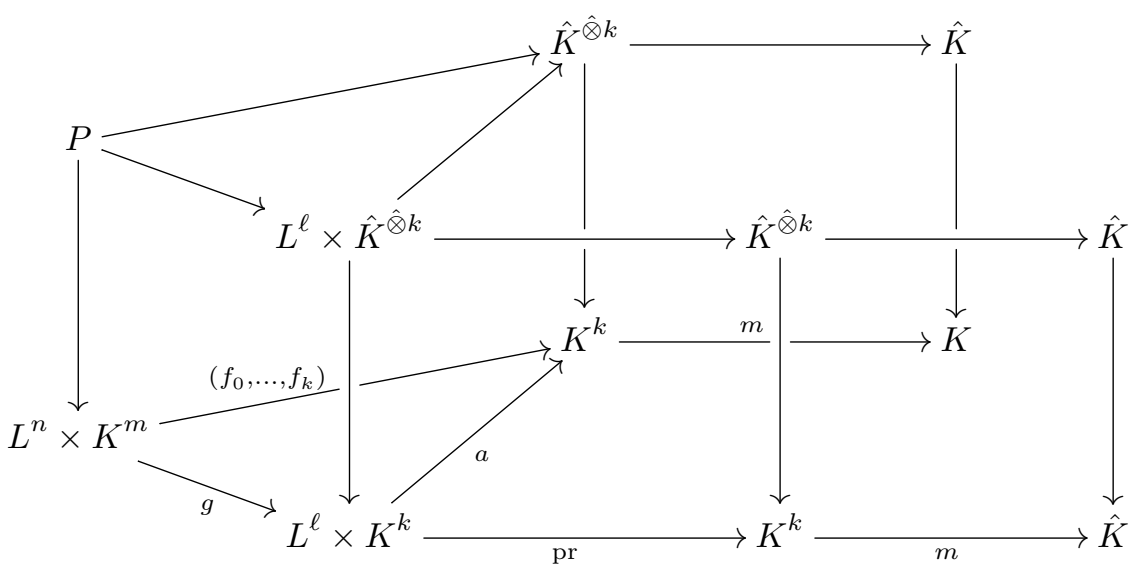

This means that an $\mathrm{xm}$-bundle $P$ given by a map $f$ is isomorphic (via a unique isomorphism) to a bundle pulled back by only the structure maps that do not include Ad (i.e. along the bottom sequence of arrows in the diagram above). Now suppose that we have two xm-bundles $P$ and $Q$, with an $\mathrm{xm}$-morphism between them. Then if we write them in the reduced form above (by which we mean they are pullbacks by maps not involving the adjoint action) there will be a unique $\mathrm{xm}$-morphism between them. Since there is a unique isomorphism from $P$ to its reduced form and from $Q$ to its reduced form, we have a unique xm-morphism from $P$ to $Q$.

What Lemma 6.4 means is that if we have two $\mathrm{xm}$-bundles and we write out the fibres of each as

$$
\hat{K}_{f_{1}\left(l_{1}, \ldots, l_{n}, k_{1}, \ldots, k_{m}\right)} \otimes \hat{K}_{f_{2}\left(l_{1}, \ldots, l_{n}, k_{1}, \ldots, k_{m}\right)} \otimes \cdots \otimes \hat{K}_{f_{k}\left(l_{1}, \ldots, l_{n}, k_{1}, \ldots, k_{m}\right)},
$$

then if the product of all the subscripts are equal once the maps involving Ad are removed, there is a unique $\mathrm{xm}$-morphism between them.

6.2. Bundle 2-gerbes and trivialisations. In [37 the third author defines a notion related to the one developed in this paper; that of a bundle 2-gerbe. The definition in [37] is quite complicated, however we can make a useful simplification by employing the ideas from Section 2, specifically Proposition 3.1. This gives us the following equivalent definition of bundle 2-gerbe:

Definition 6.5. A bundle 2-gerbe $(\mathcal{G}, P)$ on $X$ consists of the following data:

(1) a surjective submersion $P \rightarrow X$;

(2) a bundle gerbe $\mathcal{G}=(E, Y)$ over $P^{[2]}$;

(3) a trivialisation $M$ of $\left(\delta(E), \mu^{-1}(Y)_{2}\right)$; and

(4) a section $a$ of $A_{M} \rightarrow P^{[4]}$ satisfying $\delta(a)=1$ as a section of $\delta\left(A_{M}\right)$.

Here the bundle gerbe $\left(\delta(E), \mu^{-1}(Y)_{2}\right)$ is the restriction of $\delta(\mathcal{G})$ to the surjective submersion $\mu^{-1}(Y)_{2} \rightarrow P^{[3]}$ as in Example 2.9, and the line bundle $A_{M} \rightarrow P^{[4]}$ is the descent of $\delta(M) \rightarrow \mu^{-1}(Y)_{2}$ as in Proposition 3.1

Remark 6.3. We leave it to the reader to show that this definition is equivalent to the one in [37. The main point is that the definition from [37] involves a trivialisation $M$ of the bundle gerbe $(\delta(E), \delta(Y))$, and so $\delta(M) \rightarrow \delta^{2}(Y)$ does not descend as in our definition (since the bundle gerbe $\delta(\delta(E), \delta(Y))=\left(\delta^{2}(E), \delta^{2}(Y)\right)$ is not strongly trivial). Therefore one needs to consider the difference of $\delta(M)$ and the canonical trivialisation of $\left(\delta^{2}(E), \delta^{2}(Y)\right)$. One then has a section of this and the appropriate conditions on this section. 
Specifically, we are interested in trivial bundle 2-gerbes. With the appropriate modifications the definition of a trivialisation of a bundle 2-gerbe is

Definition 6.6. A bundle 2-gerbe $(\mathcal{G}, P)$ over $X$ is trivial if the following conditions are satisfied:

(1) there exists a bundle gerbe $\mathcal{H}=(Q, Z)$ over $P$ and a stable isomorphism $L: \mathcal{G} \rightarrow$ $\delta(\mathcal{H})$, where $\delta(\mathcal{H})=\left(\delta(Q), \mu^{-1}\left(Z^{2}\right)\right) ;$

(2) there exists a section $\theta$ of the bundle $M \otimes \delta(L)$ over $P^{[3]}$ satisfying $\delta(\theta)=a$.

The data $(\mathcal{H}, L, \theta)$ will be called a trivialisation of the bundle 2-gerbe.

Note that if $\mathcal{G}=(E, Y)$ then in $(1)$ the bundle $L$ sits over the space $Y \times_{P^{[2]}} \mu^{-1}\left(Z^{2}\right)$ and in $(2) M \otimes \delta(L)$ sits over the space $\mu^{-1}(Y)_{2} \times{ }_{P^{[3]}} \mu^{-1}\left(Z^{3}\right)$ but descends to $P^{[3]}$. The equation $\delta(\theta)=a$ makes sense because $\delta(\theta)$ is a section of $\delta(M \otimes \delta(L))=\delta(M)$, viewed as a bundle over $P^{[4]}$, which is the bundle $A_{M}$.

6.3. The Chern-Simons bundle 2-gerbe. The example of a bundle 2-gerbe in which we are interested is the Chern-Simons bundle 2-gerbe [11 associated to a principal Gbundle $P \rightarrow X$. This is defined by taking the simplicial manifold $P^{[\bullet+1]}$ and using the isomorphism $P^{[2]}=P \times G$ to pull back the basic gerbe on $G$ to $P^{[2]}$. The model of the basic bundle gerbe that we use here is different to that in Section 5 ; it is the lifting bundle gerbe for the path fibration of the group $G$. The lifting bundle gerbe was introduced in 32 and the example of the path fibration of a compact Lie group $G$ was studied in detail in 33. It is given by taking the surjective submersion $P G \rightarrow G$, which is a principal $\Omega G$ bundle, and identifying $P G^{[2]}$ with $P G \times \Omega G$. We then pull back the central extension $\widehat{\Omega G}$ by the projection $P G \times \Omega G \rightarrow \Omega G$.

Next we give the data of the Chern-Simons bundle 2-gerbe in detail. According to the description above, it is the pullback of the basic gerbe on $G$. Thus we have the following depiction.

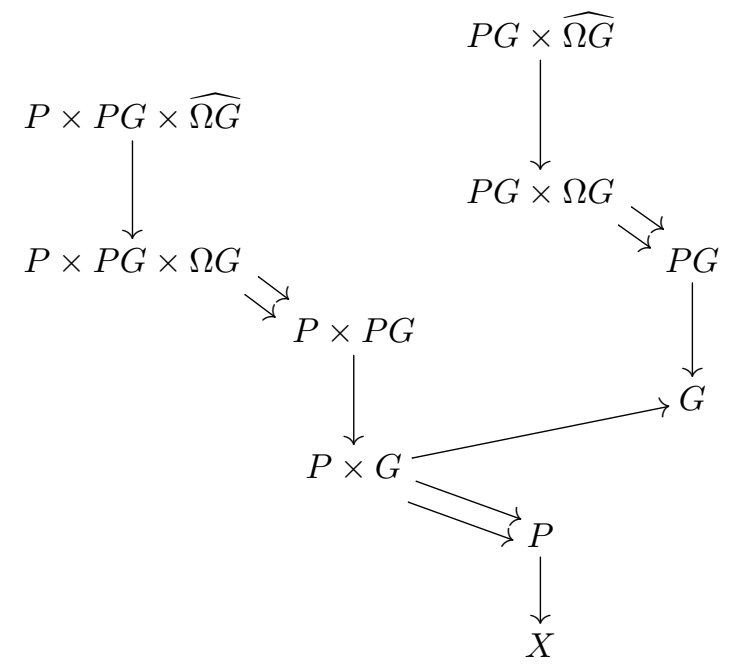

We shall denote the bundle gerbe over $P \times G$ by $\mathcal{G}$.

In order to describe the rest of the data defining the Chern-Simons bundle 2-gerbe as per Definition 6.5. we need to consider the semi-simplicial surjective submersion $\mu^{-1}(P \times$ $P G) \bullet \rightarrow P^{[\bullet+1]}$ and the bundle gerbe

$$
\delta(\mathcal{G})=\left(\delta(P \times P G \times \widehat{\Omega G}), \mu^{-1}(P \times P G)_{2}\right) .
$$


Calculation shows that the low dimensional spaces in the semi-simplicial surjective submersion $\mu^{-1}(P \times P G) \bullet \rightarrow P^{[\bullet+1]}$ are

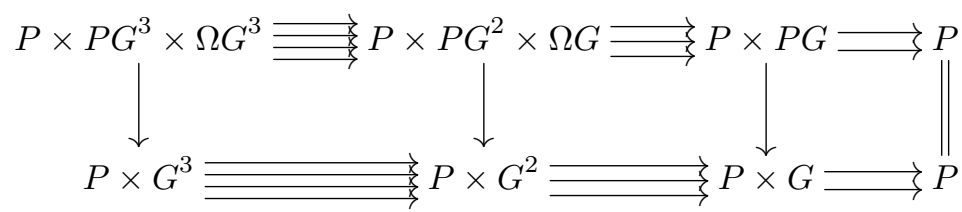

The maps $d_{i}: \mu^{-1}(P \times P G)_{2}=P \times P G^{2} \times \Omega G \rightarrow P \times P G$ are given by

$$
\begin{aligned}
& d_{0}\left(p, \gamma_{1}, \gamma_{2}, \omega\right)=\left(p \gamma_{1}(1), \gamma_{2}\right), \\
& d_{1}\left(p, \gamma_{1}, \gamma_{2}, \omega\right)=\left(p, \gamma_{1} \gamma_{2} \omega\right), \\
& d_{2}\left(p, \gamma_{1}, \gamma_{2}, \omega\right)=\left(p, \gamma_{1}\right),
\end{aligned}
$$

and the maps $d_{i}: \mu^{-1}(P \times P G)_{3}=P \times P G^{3} \times \Omega G^{3} \rightarrow P \times P G^{2} \times \Omega G$ are given by

$$
\begin{aligned}
& d_{0}\left(p, \gamma_{1}, \gamma_{2}, \gamma_{3}, \omega_{1}, \omega_{2}, \omega_{3}\right)=\left(p \gamma_{1}(1), \gamma_{2}, \gamma_{3}, \omega_{3}\right), \\
& d_{1}\left(p, \gamma_{1}, \gamma_{2}, \gamma_{3}, \omega_{1}, \omega_{2}, \omega_{3}\right)=\left(p, \gamma_{1} \gamma_{2} \omega_{1}, \gamma_{3}, \operatorname{Ad}_{\gamma_{3}^{-1}}\left(\omega_{1}^{-1}\right) \omega_{2}\right), \\
& d_{2}\left(p, \gamma_{1}, \gamma_{2}, \gamma_{3}, \omega_{1}, \omega_{2}, \omega_{3}\right)=\left(p, \gamma_{1}, \gamma_{2} \gamma_{3} \omega_{3}, \omega_{3}^{-1} \omega_{2}\right), \\
& d_{3}\left(p, \gamma_{1}, \gamma_{2}, \gamma_{3}, \omega_{1}, \omega_{2}, \omega_{3}\right)=\left(p, \gamma_{1}, \gamma_{2}, \omega_{1}\right) .
\end{aligned}
$$

Note that

$$
\mu^{-1}(P \times P G)_{2}^{[2]}=\left(P \times P G^{2} \times \Omega G\right) \times{ }_{P \times G^{2}}\left(P \times P G^{2} \times \Omega G\right)=P \times P G^{2} \times \Omega G^{4}
$$

via the projections $\left(p, \gamma_{1}, \gamma_{2}, \omega_{0}, \omega_{1}, \omega_{2}, \omega_{3}\right) \mapsto\left(p, \gamma_{1}, \gamma_{2}, \omega_{0}\right)$ and $\left(p, \gamma_{1} \omega_{1}, \gamma_{2} \omega_{2}, \omega_{3}\right)$. The maps $\mu^{-1}(P \times P G)_{2}^{[2]}=P \times P G^{2} \times \Omega G^{4} \rightrightarrows P \times P G \times \Omega G=(P \times P G)^{[2]}$ are given by

$$
\begin{aligned}
& d_{0}\left(p, \gamma_{1}, \gamma_{2}, \omega_{0}, \omega_{1}, \omega_{2}, \omega_{3}\right)=\left(p \gamma_{1}(1), \gamma_{2}, \omega_{2}\right), \\
& d_{1}\left(p, \gamma_{1}, \gamma_{2}, \omega_{0}, \omega_{1}, \omega_{2}, \omega_{3}\right)=\left(p, \gamma_{1} \gamma_{2} \omega_{0}, \omega_{0}^{-1} \operatorname{Ad}_{\gamma_{2}^{-1}}\left(\omega_{1}\right) \omega_{2} \omega_{3}\right), \\
& d_{2}\left(p, \gamma_{1}, \gamma_{2}, \omega_{0}, \omega_{1}, \omega_{2}, \omega_{3}\right)=\left(p, \gamma_{1}, \omega_{1}\right) .
\end{aligned}
$$

Therefore, the bundle gerbe $\delta(\mathcal{G})$ is given by the line bundle $E \rightarrow P \times P G^{2} \times \Omega G^{4}$ whose fibre at $\left(p, \gamma_{1}, \gamma_{2}, \omega_{0}, \omega_{1}, \omega_{2}, \omega_{3}\right)$ is

$$
\begin{aligned}
E_{\left(p, \gamma_{1}, \gamma_{2}, \omega_{0}, \omega_{1}, \omega_{2}, \omega_{3}\right)} & \simeq \widehat{\Omega G}_{\omega_{2}} \otimes \widehat{\Omega G}_{\omega_{0}^{-1} \mathrm{Ad} \gamma_{\gamma_{2}}\left(\omega_{1}\right) \omega_{2} \omega_{3}}^{*} \otimes \widehat{\Omega G}_{\omega_{1}} \\
& \simeq \widehat{\Omega G}_{\omega_{2}} \otimes \widehat{\Omega G}_{\omega_{0}^{-1}}^{*} \otimes \widehat{\Omega G}_{\mathrm{Ad}_{\gamma_{2}^{-1}}\left(\omega_{1}\right)}^{*} \otimes \widehat{\Omega G}_{\omega_{2}}^{*} \otimes \widehat{\Omega G}_{\omega_{3}}^{*} \otimes \widehat{\Omega G}_{\omega_{1}} \\
& \simeq \widehat{\Omega G}_{\omega_{2}} \otimes \widehat{\Omega G}_{\omega_{0}} \otimes \widehat{\Omega G}_{\omega_{1}^{-1}} \otimes \widehat{\Omega G}_{\omega_{2}^{-1}} \otimes \widehat{\Omega G}_{\omega_{3}^{-1}} \otimes \widehat{\Omega G}_{\omega_{1}} \\
& \simeq \widehat{\Omega G}_{\omega_{0}} \otimes \widehat{\Omega G}_{\omega_{3}^{-1}} .
\end{aligned}
$$

The isomorphisms above are the unique ones guaranteed by Lemma 6.4 This calculation shows that the bundle gerbe $\delta(\mathcal{G})$ is trivial with trivialisation $M=P \times P G^{2} \times \widehat{\Omega G}^{*} \rightarrow$ $P \times P G^{2} \times \Omega G$, which is the data of Definition 6.5 (3).

For 6.5 (4) we note that the bundle $\delta(M) \rightarrow P \times P G^{3} \times \Omega G^{3}$ has fibre at the point $\left(p, \gamma_{1}, \gamma_{2}, \gamma_{3}, \omega_{1}, \omega_{2}, \omega_{3}\right)$ given by

$$
\widehat{\Omega G}_{\omega_{3}}^{*} \otimes \widehat{\Omega G}_{\mathrm{Ad}_{\gamma_{3}-1}\left(\omega_{1}^{-1}\right) \omega_{2}} \otimes \widehat{\Omega G}_{\omega_{3}^{-1} \omega_{2}}^{*} \otimes \widehat{\Omega G}_{\omega_{1}}
$$


and so we have the (unique) sequence of isomorphisms

$$
\begin{aligned}
\delta(M)_{\left(p, \gamma_{1}, \gamma_{2}, \gamma_{3}, \omega_{1}, \omega_{2}, \omega_{3}\right)} & \simeq \widehat{\Omega G}_{\omega_{3}}^{*} \otimes \widehat{\Omega G}_{\mathrm{Ad}_{\gamma_{3}^{-1}}\left(\omega_{1}^{-1}\right) \omega_{2}} \otimes \widehat{\Omega G}_{\omega_{3}^{-1} \omega_{2}}^{*} \otimes \widehat{\Omega G}_{\omega_{1}} \\
& \simeq \widehat{\Omega G}_{\omega_{3}}^{*} \otimes \widehat{\Omega G}_{\mathrm{Ad}_{\gamma_{3}^{-1}}\left(\omega_{1}^{-1}\right)} \otimes \widehat{\Omega G}_{\omega_{2}} \otimes \widehat{\Omega G}_{\omega_{3}^{-1}}^{*} \otimes \widehat{\Omega G}_{\omega_{2}}^{*} \otimes \widehat{\Omega G}_{\omega_{1}} \\
& \simeq \widehat{\Omega G}_{\omega_{3}}^{*} \otimes \widehat{\Omega G}_{\omega_{1}^{-1}} \otimes \widehat{\Omega G}_{\omega_{2}} \otimes \widehat{\Omega G}_{\omega_{3}^{-1}}^{*} \otimes \widehat{\Omega G}_{\omega_{2}}^{*} \otimes \widehat{\Omega G}_{\omega_{1}} \\
& \simeq \widehat{\Omega G}_{\omega_{3}}^{*} \otimes \widehat{\Omega G}_{\omega_{1}}^{*} \otimes \widehat{\Omega G}_{\omega_{2}} \otimes \widehat{\Omega G}_{\omega_{3}} \otimes \widehat{\Omega G}_{\omega_{2}}^{*} \otimes \widehat{\Omega G}_{\omega_{1}} \\
& \simeq U(1) .
\end{aligned}
$$

Therefore we have a trivialisation of $\delta(M)$ and hence $A_{M}$, by Lemma 6.4. We define the section $a$ from Definition 6.5 (4) to be this trivialisation.

The situation is summarised in the following diagram

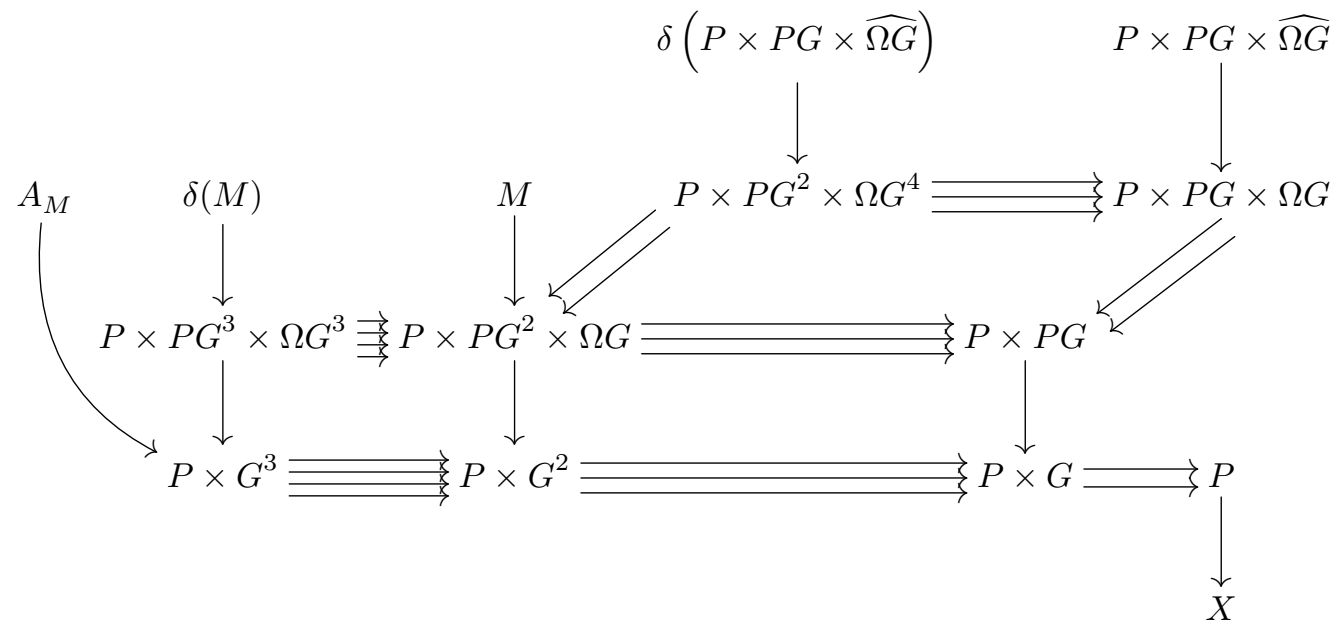

6.4. The simplicial extension of a string structure. Suppose now that we have a trivialisation $\mathcal{H}$ of the Chern-Simons bundle 2-gerbe associated to the $G$-bundle $P \rightarrow X$. We will build a simplicial extension of $\mathcal{H}$ over the simplicial manifold $E \mathcal{K}(P)$. associated to the action of the crossed module $\mathcal{K}=(\widehat{\Omega G} \rightarrow P G)$ from Example 6.1 on $P$. We will now describe this simplicial manifold in more detail.

Given a crossed module $\hat{K} \stackrel{t}{\rightarrow} L$ of Lie groups acting on a manifold $P$, one can form an action 2-groupoid. This has as objects the manifold $P$, as 1 -arrows the product $P \times L$, and as 2-arrows the product $P \times L \times \hat{K}$ :

$$
P \times L \times \hat{K} \underset{1_{P} \times f}{\stackrel{\mathrm{pr}_{12}}{\longrightarrow}} P \times L \underset{\text { act }}{\stackrel{\mathrm{pr}_{1}}{\longrightarrow}} P
$$

where the action $f$ of $\hat{K}$ on $L$ is via the map $t$ and the action of $L$ on $P$ is part of the definition of the action of $\mathcal{K}$ on $P$. The precise description of the structural maps (i.e. sources, targets and compositions) of this 2-groupoid we shall leave to the reader as an instructive exercise, since we are more interested in the nerve of this 2-groupoid (as defined by Street [40] and Duskin [15]), which we shall describe explicitly in low dimensions. This nerve is what we have called $E \mathcal{K}(P)$.

The intuitive picture that the reader should keep in mind is that of the nerve of the action 1-groupoid, but instead of commuting triangles making up the dimension 2 faces of simplices, one should fill it with an element of the group $\hat{K}$. A 2-simplex is thus a triangle commuting up to a 2-arrow; a 3-simplex is a tetrahedron with faces labelled as such as commuting in the 2-dimensional sense. Table 1 specifies the face maps that we shall need in the course of this section. 


\begin{tabular}{|c|c|}
\hline$E \mathcal{K}(P)_{1} \rightrightarrows E \mathcal{K}(P)_{0}$ & $\begin{array}{l}d_{0}(p, l)=p l \\
d_{1}(p, l)=p\end{array}$ \\
\hline$E \mathcal{K}(P)_{2} \rightrightarrows E \mathcal{K}(P)_{1}$ & $\begin{array}{l}d_{0}\left(p, l_{1}, l_{2}, k\right)=\left(p l_{1}, l_{2}\right) \\
d_{1}\left(p, l_{1}, l_{2}, k\right)=\left(p, l_{1} l_{2} t(k)\right) \\
d_{2}\left(p, l_{1}, l_{2}, k\right)=\left(p, l_{1}\right)\end{array}$ \\
\hline$E \mathcal{K}(P)_{3} \Longrightarrow$ & $\begin{array}{l}d_{0}\left(p, l_{1}, l_{2}, l_{3}, k_{1}, k_{2}, k_{3}\right)=\left(p l_{1}, l_{2}, l_{3}, k_{3}\right) \\
d_{1}\left(p, l_{1}, l_{2}, l_{3}, k_{1}, k_{2}, k_{3}\right)=\left(p, l_{1} l_{2} t\left(k_{1}\right), l_{3}, k_{2}\right) \\
d_{2}\left(p, l_{1}, l_{2}, l_{3}, k_{1}, k_{2}, k_{3}\right)=\left(p, l_{1}, l_{2} l_{3} t\left(k_{3}\right), k_{3}^{-1}\left(k_{1}^{l^{-1}}\right) k_{2}\right) \\
d_{3}\left(p, l_{1}, l_{2}, l_{3}, k_{1}, k_{2}, k_{3}\right)=\left(p, l_{1}, l_{2}, k_{1}\right)\end{array}$ \\
\hline
\end{tabular}

TABLE 1. The nerve of the action 2-groupoid in low dimensions

The crossed module we are interested in is $\widehat{\Omega G} \rightarrow P G$, from Example 6.1, which gives rise to the String group of $G$. As described earlier, it acts naturally on the $G$-bundle $P$ via the map to $G$. For the simplicial manifold $E \mathcal{K}(P)$. arising from this action there is a simplicial map $e: E \mathcal{K}(P) . \rightarrow P^{[\bullet+1]}$, given by evaluating all paths at their endpoints, and forgetting factors of $\widehat{\Omega G}$. In low degrees this is

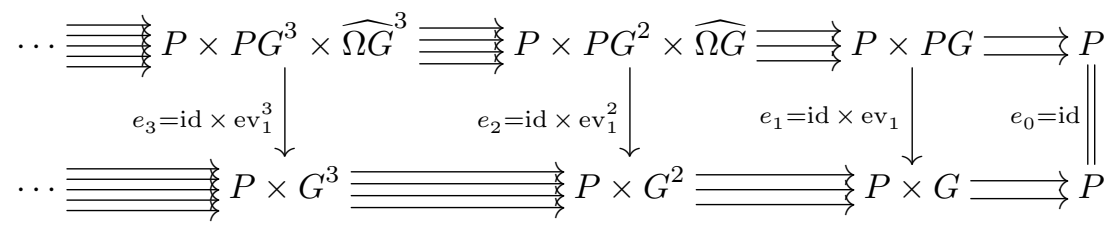

We shall denote the operation $\delta$ for the simplicial manifolds $E \mathcal{K}(P) \bullet$ and $P^{[\bullet+1]}$ by $\delta_{E \mathcal{K}}$ and $\delta_{P}$, respectively. Thus the definition of the Chern-Simons bundle 2-gerbe and a trivialisation of it involve $\delta_{P}$ everywhere, while a simplicial extension of $\mathcal{H}$ over $E \mathcal{K}(P)$. will involve $\delta_{E \mathcal{K}}$. We also remind the reader that if $\mathcal{G}=(E, Y)$ over $P^{[2]}$ and $\mathcal{H}=(Q, Z)$ over $P$ then by $\delta^{k}(\mathcal{G})$ we mean $\left(\delta^{k}(E), \mu^{-1}(Y)_{k}\right)$ and by $\delta^{k}(\mathcal{H})$ we mean $\left(\delta^{k}(Q), \mu^{-1}\left(Z^{k+1}\right)\right)$.

Given a trivialisation $(\mathcal{H}, L, \theta)$ of the Chern-Simons bundle 2 -gerbe, we will construct a simplicial extension of $\mathcal{H}$ over $E \mathcal{K}(P)$. by pulling back the data of the bundle 2 -gerbe $(\mathcal{G}, P)$ along $e$.

We construct the trivialisation $T$ of $\delta_{E \mathcal{K}}(\mathcal{H})$ as follows. Notice first, since $e$ is a simplicial map, we have that $e^{-1}\left(\delta_{P}(\mathcal{H})\right)$ is canonically isomorphic to $\delta_{E \mathcal{K}}(\mathcal{H})$. We therefore have the stable isomorphism $e_{1}^{-1}(L): e_{1}^{-1}(\mathcal{G}) \rightarrow e_{1}^{-1}\left(\delta_{P}(\mathcal{H})\right)=\delta_{E \mathcal{K}}(\mathcal{H})$. To construct $T$ we combine this with a trivialisation of $e_{1}^{-1}(\mathcal{G})$ using the following lemma.

Lemma 6.7. Let $(Q, Y)$ be a bundle gerbe over a manifold $X$ with surjective submersion $\pi: Y \rightarrow X$. Then $\pi^{-1}(Q, Y)$ (as a bundle gerbe over $Y$ ) has a canonical trivialisation given by $\tau=Q$.

Proof. First notice that the statement makes sense because $\pi^{-1}(Q, Y)$ has as surjective submersion the pullback $\pi^{-1}(Y)=Y^{[2]}$, and $\tau=Q$ is a line bundle over $Y^{[2]}$. The fibre product $Y^{[2]} \times_{Y} Y^{[2]}$ is given by $Y^{[3]}$ and the face maps are projection onto the first 
and second, and first and third factors, respectively. Thus we have $\pi^{-1}(Q)_{\left(y_{1}, y_{2}, y_{3}\right)}=$ $Q_{\left(y_{2}, y_{3}\right)}=Q_{\left(y_{2}, y_{1}\right)} Q_{\left(y_{1}, y_{3}\right)}=Q_{\left(y_{1}, y_{2}\right)}^{*} Q_{\left(y_{1}, y_{3}\right)}$, and so $\pi^{-1}(Q)$ is trivialised by $\tau=Q$.

Now, $e_{1}: P \times P G \rightarrow P \times G$ is the surjective submersion for the bundle gerbe $\mathcal{G}$, so Lemma 6.7 gives us the trivialisation $\tau$ of $e_{1}^{-1}(\mathcal{G})$. Thus we have the trivialisation $T=\tau \otimes e_{1}^{-1}(L)$ of $\delta_{E \mathcal{K}}(\mathcal{H})$.

Next we need a section $s$ of $A_{T}=\delta_{E \mathcal{K}}\left(\tau \otimes e_{1}^{-1}(L)\right)$ over $E \mathcal{K}(P)_{2}=P \times P G^{2} \times \widehat{\Omega G}$. Since $\mathcal{H}$ is a trivialisation of the Chern-Simons bundle 2 -gerbe we have a section $\theta$ of $M \otimes \delta_{P}(L)$ over $P \times G^{2}$. We claim that $e_{2}^{-1}\left(M \otimes \delta_{P}(L)\right)$ is canonically isomorphic to $\delta_{E \mathcal{K}}\left(\tau \otimes e_{1}^{-1}(L)\right)$ and therefore we can define $s=e_{2}^{-1}(\theta)$. We have

$$
e_{2}^{-1}\left(M \otimes \delta_{P}(L)\right)=e_{2}^{-1}(M) \otimes e_{2}^{-1}\left(\delta_{P}(L)\right)=e_{2}^{-1}(M) \otimes \delta_{E \mathcal{K}}\left(e_{1}^{-1}(L)\right) .
$$

So we need to show that $e_{2}^{-1}(M)$ is canonically isomorphic to $\delta_{E \mathcal{K}}(\tau)$. Notice that $P \times P G^{2} \times \widehat{\Omega G}$ is the total space of the dual of $M$. We have the following result.

Lemma 6.8. Let $(Q, Y)$ be a bundle gerbe on a manifold $X$, with surjective submersion $\pi: Y \rightarrow X$. Let $\hat{\pi}: R \rightarrow Y$ be a trivialisation of $(Q, Y)$, and $R^{*}$ its dual. Denote by $p$ the composite map $\pi \circ \hat{\pi}: R^{*} \rightarrow Y \rightarrow X$. Recall that, by Lemma 6. 7 , the bundle gerbe $\pi^{-1}(Q, Y)$ has a trivialisation $\tau$. Then the two trivialisations $p^{-1}(R)$ and $\hat{\pi}^{-1}\left(\tau^{*}\right)$ of $p^{-1}(Q, Y)$ are canonically isomorphic.

Proof. Recall from the proof of Lemma 6.7 that the bundle gerbe $\pi^{-1}(Q, Y)$ over $Y$ has as surjective submersion $Y^{[2]} \rightarrow Y$. It has two trivialisations; $\tau$ and $\pi^{-1}(R)$. We have $\tau \oslash \pi^{-1}(R)=R^{*}$ since, for $y \in Y$ and any $x$ in the same fibre, $\left(\tau \otimes \pi^{-1}(R)^{*}\right)_{(y, x)}=$ $Q_{(y, x)} R_{x}=R_{y}^{*}$, using the fact that $R$ is a trivialisation. We have $\hat{\pi}^{-1}(\tau) \oslash p^{-1}(R)=$ $\hat{\pi}^{-1}\left(\tau \oslash \pi^{-1}(R)\right)=\hat{\pi}^{-1}\left(R^{*}\right)$, which is canonically trivial. Therefore $\hat{\pi}^{-1}(\tau)$ is canonically isomorphic to $p^{-1}(R)$.

To apply Lemma 6.8 to the bundle gerbe $\delta_{P}(\mathcal{G})$ with its trivialisation $M$ notice that $e_{2}$ factors as $P \times P G^{2} \times \widehat{\Omega G} \stackrel{\hat{\pi}}{\rightarrow} P \times P G^{2} \times \Omega G=\mu^{-1}(P \times P G)_{2} \stackrel{\pi}{\rightarrow} P \times G^{2}$ such that the following diagram commutes

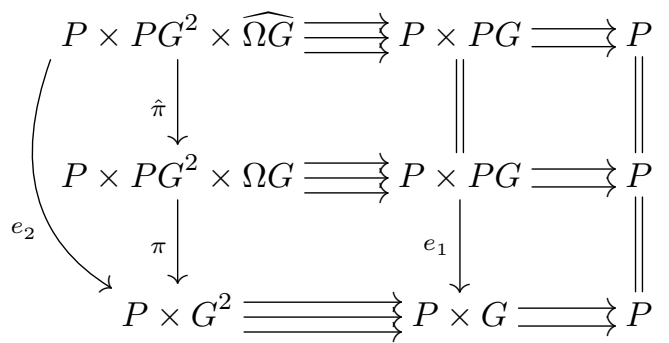

and so the trivialisation $e_{2}^{-1}(M)$ is canonically isomorphic to $\hat{\pi}^{-1}\left(\tau_{\delta(\mathcal{G})}\right)$, where $\tau_{\delta(\mathcal{G})}$ is the canonical trivialisation of $\pi^{-1}\left(\delta_{P}(\mathcal{G})\right)$ given by Lemma 6.7. But $\tau_{\delta(\mathcal{G})}$ is isomorphic to $\delta_{P}(\tau)$, where $\tau$ is the canonical trivialisation of $e_{1}^{-1}(\mathcal{G})$. Hence the pullback $\hat{\pi}^{-1}\left(\tau_{\delta(\mathcal{G})}\right)=$ $\hat{\pi}^{-1}\left(\delta_{P}(\tau)\right)$ is isomorphic to $\delta_{E \mathcal{K}}$ applied to the dual of the canonical trivialisation of $e_{1}^{-1}(\mathcal{G})$, which is precisely $\delta_{E \mathcal{K}}(\tau)$. This allows us to define the section $s$ as the pullback of $\theta$ by $e_{2}$.

It only remains to show that $\delta_{E \mathcal{K}}(s)=1$ as a section of $\delta_{E \mathcal{K}}\left(A_{T}\right)$, which is the descent of the bundle $\delta_{E \mathcal{K}}^{2}\left(\tau \otimes e_{1}^{-1}(L)\right)$ to $E \mathcal{K}(P)_{3}=P \times P G^{3} \times \widehat{\Omega G}^{3}$. Notice that since $(\mathcal{H}, L, \theta)$ is a trivialisation of the Chern-Simons bundle 2-gerbe we have $\delta_{P}(\theta)=a$, where $a$ is the section of $A_{M}$ from Section 6.3. Further, since $s=e_{2}^{-1}(\theta)$, we have $\delta_{E \mathcal{K}}(s)=\delta_{E \mathcal{K}}\left(e_{2}^{-1}(\theta)\right)=e_{3}^{-1}\left(\delta_{P}(\theta)\right)=e_{3}^{-1}(a)$. Therefore, we need only show that $e_{3}^{-1}(a)$ is the canonical trivialisation of $\delta_{E \mathcal{K}}^{2}\left(\tau \otimes e_{1}^{-1}(L)\right)$ under the isomorphism induced by Lemma 6.8 . In fact, since $a$ is a trivialisation of $A_{M}=\delta_{P}(M)=\delta_{P}(M) \otimes \delta_{P}^{2}(L)$, the 
section $\delta_{P}(\theta)$ induces the canonical trivialisation of $\delta_{P}^{2}(L)$ and so it suffices to check that $e_{3}^{-1}(a)$ induces the canonical trivialisation of $\delta_{E \mathcal{K}}^{2}(\tau)$. Notice however, that the canonical trivialisation of $\delta_{E \mathcal{K}}^{2}(\tau)$ involves pairing up factors of $\widehat{\Omega G}$ and $\widehat{\Omega G}^{*}$, whereas $a$ is an $\mathrm{xm}$ morphism. To see that these are the same consider a crossed module $\hat{K} \stackrel{t}{\rightarrow} L$. We can factorize the constant map $K \rightarrow K ; x \mapsto 1$ as

$$
K \stackrel{\Delta}{\rightarrow} K \times K \stackrel{1 \times i}{\longrightarrow} K \times K \stackrel{m}{\rightarrow} K,
$$

where $\Delta$ is the diagonal map, and $i$ and $m$ are inversion and multiplication in $K$, respectively. Then $(m \circ(1 \times i) \circ \Delta)^{-1}(K)$ is canonically trivial. However, we also have that $(m \circ(1 \times i) \circ \Delta)^{-1}(K)$ is isomorphic to $\hat{K} \otimes \hat{K}^{*}$, which is canonically trivial. We have the following trivial result

Lemma 6.9. The two trivialisations of $\hat{K} \otimes \hat{K}^{*}$ given above are equal.

The point is that both the canonical trivialisation of $\delta_{E \mathcal{K}}^{2}(\tau)$ (by Lemma 6.9) and the trivialisation given by $e_{3}^{-1}(a)$ are $\mathrm{xm}$-morphisms as in Definition 6.3, and therefore are equal by Lemma 6.4. So Lemma 6.9 tells us that the section $e_{3}^{-1}(a)$ (and hence $\delta_{E \mathcal{K}}(s)$ ) agrees with the canonical section of $\delta_{E \mathcal{K}}^{2}\left(\tau \otimes e_{1}^{-1}(L)\right)$ (and hence $\delta_{E \mathcal{K}}\left(A_{T}\right)$ ). Therefore we have our main result

Theorem 6.10. Let $P \rightarrow X$ be a principal $G$-bundle and let $(\mathcal{H}, L, \theta)$ be a trivialisation of the Chern-Simons bundle 2-gerbe of $P$. Then $\mathcal{H}$ has a simplicial extension over the nerve of the action 2-groupoid of the induced String group action on $P$, given by $(T, s)$ constructed above.

\section{Appendix A. Descent For trivialisations}

Proposition A.1. Assume that $(P, Y)$ is a bundle gerbe over $M$ and that $\phi: X \rightarrow Y$ is morphism of surjective submersions over $M$. Then if $T \rightarrow Y$ is a trivialisation of $\left(\phi^{-1}(P), X\right)$ there is a trivialisation $\phi(T) \rightarrow Y$ with the property that $\phi^{-1}(\phi(T)) \rightarrow X$ is isomorphic to $T$ as a trivialisation of $\left(\phi^{-1}(P), Y\right)$.

Proof. Recall that a trivialisation $R \rightarrow Y$ of $(P, Y)$ is an isomorphism $P \rightarrow \delta_{Y}(R)$ which commutes with the bundle gerbe product on $P$ and the trivial bundle gerbe product on $\delta_{Y}(R)$. It is convenient to formulate this in the following way. For $\left(y_{1}, y_{2}\right) \in Y^{[2]}$ we have an isomorphism

$$
\begin{aligned}
& R_{y_{1}} \otimes P_{\left(y_{1}, y_{2}\right)} \rightarrow R_{y_{2}} \\
& r_{1} \otimes p_{12} \mapsto r_{1} p_{12}
\end{aligned}
$$

and we require that for any $y_{1}, y_{2}, y_{3}$ we have $\left(r_{1} p_{12}\right) p_{23}=r_{1}\left(p_{12} p_{23}\right)$ where $p_{i j} \in P_{\left(y_{i}, y_{j}\right)}$ and $\left(p_{12} p_{23}\right)$ denotes the bundle gerbe product.

So if $T \rightarrow X$ is a trivialisation of $\phi^{-1}(P) \rightarrow X^{[2]}$ then we have

$$
T_{x_{1}} P_{\left(\phi\left(x_{1}\right), \phi\left(x_{2}\right)\right)} \rightarrow T_{x_{2}},
$$

with the corresponding condition on compatibility with the bundle gerbe product. We define $S \rightarrow X \times_{M} Y$ by $S_{(x, y)}=T_{x} \otimes P_{(\phi(x), y)}$. We want to show that $S$ descends to a bundle $\phi(T) \rightarrow Y$ and to this end we define

$$
\phi_{x_{2} x_{1}}: S_{x_{1}, y} \rightarrow S_{x_{2}, y}
$$

by $\phi_{x_{2} x_{1}}\left(t_{1} \otimes q_{1}\right)=\left(t_{1} p_{12}\right) \otimes\left(p_{12}^{*} q_{1}\right)$ where $t_{1} \in T_{x_{1}}, q_{1} \in P_{(\phi(x), y)}$ and the definition involves the choice of $p_{12} \in P_{\left(\phi\left(x_{1}\right), \phi\left(x_{2}\right)\right)}$. It is clearly independent of this choice and the choices representing the element in $S_{\left(x_{1}, y\right)}$. We need to check that $\phi_{x_{3} x_{2}} \phi_{x_{2} x_{1}}=\phi_{x_{3} x_{1}}$ 
and making appropriate choices of elements in the various spaces we have

$$
\begin{aligned}
\phi_{x_{3} x_{2}} \phi_{x_{2} x_{1}}\left(t_{1} \otimes q_{1}\right) & =\phi_{x_{3} x_{2}}\left(t_{1} p_{12}\right) \otimes\left(p_{12}^{*} q_{1}\right) \\
& =\left(t_{1} p_{12}\right) p_{23} \otimes p_{23}^{*}\left(p_{12}^{*} q_{1}\right) \\
& =\phi_{x_{3} x_{1}}\left(t_{1} \otimes q_{1}\right)
\end{aligned}
$$

as required.

Now define

$$
\rho_{x y_{1} y_{2}}: S_{\left(x, y_{1}\right)} \otimes P_{\left(y_{1}, y_{2}\right)} \rightarrow S_{\left(x, y_{2}\right)}
$$

by $\rho_{x y_{1} y_{2}}\left(s_{1} \otimes q_{12}\right)=t_{1} \otimes q_{1} q_{12}$ where $s_{1}=t_{1} \otimes q_{1}$. We want to show that

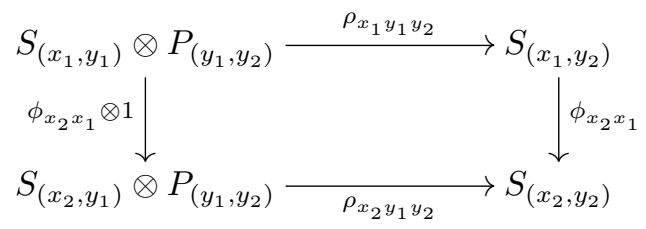

commutes. To see this we note that if $s_{1}=t_{1} \otimes q_{1}$ then

$$
\begin{aligned}
\rho_{x_{2} y_{1} y_{2}} \circ\left(\phi_{x_{2} x_{1}} \otimes 1\right)\left(s_{1} \otimes q_{12}\right) & =\rho_{x_{2} y_{1} y_{2}}\left(\left(t_{1} p_{12} \otimes p_{12}^{*} q_{1}\right) \otimes q_{12}\right) \\
& =t_{1} p_{12} \otimes\left(\left(p_{12}^{*} q_{1}\right) q_{12}\right) \\
& =t_{1} p_{12} \otimes p_{12}^{*}\left(q_{1} q_{12}\right) \\
& =\phi_{\left(x_{2} x_{1}\right)}\left(t_{1} \otimes\left(q_{1} q_{12}\right)\right) \\
& =\phi_{\left(x_{2} x_{1}\right)} \circ \rho_{x_{1} y_{1} y_{2}}\left(s_{1} \otimes q_{12}\right) .
\end{aligned}
$$

Hence this map descends to give an isomorphism

$$
\phi(T)_{y_{1}} \otimes P_{\left(y_{1}, y_{2}\right)} \rightarrow \phi(T)_{y_{2}}
$$

which we write as $s_{1} \otimes q_{12} \mapsto s_{1} q_{12}$ and we have to check that $\left(s_{1} q_{12}\right) q_{23}=s_{1}\left(q_{12} q_{23}\right)$. We have $\left(s_{1} q_{12}\right) q_{23}=\left(t_{1} \otimes q_{1} q_{12}\right) q_{23}=t_{1} \otimes\left(q_{1} q_{12}\right) q_{23}=t_{1} \otimes q_{1}\left(q_{12} q_{23}\right)=s_{1}\left(q_{12} q_{23}\right)$, as required.

Finally notice that the pullback of $\phi(T)$ is

$$
\phi^{-1}(\phi(T))_{x}=\phi(T)_{\phi(x)}=S_{(x, \phi(x))}=T_{x} P_{(\phi(x), \phi(x))}=T_{x},
$$

as required.

\section{Appendix B. Calculations supporting the proof of Proposition 5.2}

We prove the equation $\delta(f)-d \beta=\pi^{*}(\omega)$. Our strategy, as in 34, is to transfer the problem to the more convenient space $G / T \times Y_{T}$, where $T$ is the subgroup of diagonal matrices in $G=U(n)$ and $Y_{T}=(T \times Z) \cap Y$. Recall the canonical map $p_{Y}: G / T \times Y_{T} \rightarrow Y$ defined by $(g T,(t, z))=\left(g t g^{-1}, z\right)$. This map is $G$-equivariant, for the right action of $G$ on $Y$ by conjugation, if we make $G$ act on the right of $G / T \times Y_{T}$ by $(g T,(t, z)) \cdot h=$ $\left(h^{-1} g T,(t, z)\right)$. By Lemma 6.3 of [34], the induced map $p_{Y}^{*}: \Omega^{*}(Y) \rightarrow \Omega^{*}\left(G / T \times Y_{T}\right)$ on forms is injective. Therefore it suffices to prove that $\delta\left(p_{Y}^{*}(f)\right)=d p_{Y}^{*}(\beta)=\pi^{*}\left(p_{Y}^{*}(\omega)\right)$ in $\Omega^{2}\left(G / T \times Y_{T}^{[2]}\right)$.

Recall that we may identify a point in $G / T$ with a family of orthogonal projections $P_{1}, \ldots, P_{n}$ where $P_{i} P_{j}=0$ if $i \neq j$ and $\sum_{i} P_{1}=1$. We identify a point in $G / T \times Y_{T}$ with a triple $(P, \lambda, z)$, where $P=\left(P_{1}, \ldots, P_{n}\right)$ is a family of orthogonal projections as above, $\lambda=\left(\lambda_{1}, \ldots, \lambda_{n}\right) \in T$ with $z \neq \lambda_{i}$ for all $i$. Under this identification the right action of $G$ is $(P, \lambda, z) \cdot h=\left(h^{-1} P h, \lambda, z\right)$. We regard the $\lambda_{i}$ as the eigenvalues of a unitary matrix $g$ and the $P_{i}$ as the orthogonal projections onto the $\lambda_{i}$-eigenspace. Under this interpretation, the map $G / T \times Y_{T} \rightarrow Y$ is the map which sends

$$
(P, \lambda, z) \mapsto(g, z), \quad \text { where } g=\sum_{i=1}^{n} \lambda_{i} P_{i} .
$$


From equation (B.4) in 34 we have the following expression for the curving $p_{Y}^{*}(f)$ :

$$
p_{Y}^{*}(f)=\frac{i}{4 \pi} \sum_{i \neq k}\left(\log _{z} \lambda_{i}-\log _{z} \lambda_{k}+\frac{\lambda_{k}-\lambda_{i}}{\lambda_{k}}\right) \operatorname{tr}\left(P_{i} d P_{k} d P_{k}\right) .
$$

A little calculation yields that

$$
\delta\left(p_{Y}^{*}(f)\right)=\frac{i}{4 \pi} \sum_{i \neq k} A_{i k}\left(\operatorname{tr}\left(P_{i}\left[P_{k}, \theta_{h}\right] d P_{k}\right)+\operatorname{tr}\left(P_{i} d P_{k}\left[P_{k}, \theta_{h}\right]\right)+\operatorname{tr}\left(P_{i}\left[P_{k}, \theta_{h}\right]\left[P_{k}, \theta_{h}\right]\right)\right),
$$

where we have set $A_{i k}=\log _{z} \lambda_{i}-\log _{z} \lambda_{k}+\left(\lambda_{k}-\lambda_{i}\right) \lambda_{i}^{-1}$ and $\theta_{h}=d h h^{-1}$. Using the fact that $P_{i} P_{k}=0$ for $i \neq k$ and $d P_{k}=P_{k} d P_{k}+d P_{k} P_{k}$ we obtain

$$
\operatorname{tr}\left(P_{i}\left[P_{k}, \theta_{h}\right] d P_{k}\right)=-\operatorname{tr}\left(\theta_{h} d P_{k} P_{i}\right) \text {. }
$$

Similarly we obtain

$$
\begin{aligned}
& \operatorname{tr}\left(P_{i} d P_{k}\left[P_{k}, \theta_{h}\right]\right)=-\operatorname{tr}\left(\theta_{h} P_{i} d P_{k}\right) \\
& \operatorname{tr}\left(P_{i}\left[P_{k}, \theta_{h}\right]\left[P_{k}, \theta_{h}\right]\right)=-\operatorname{tr}\left(P_{i} \theta_{h} P_{k} \theta_{h}\right) .
\end{aligned}
$$

Hence our expression for $\delta\left(p_{Y}^{*}(f)\right)$ becomes

$$
\delta\left(p_{Y}^{*}(f)\right)=-\frac{i}{4 \pi} \sum_{i \neq k} A_{i k}\left(\operatorname{tr}\left(d P_{k} P_{i} \theta_{h}\right)+\operatorname{tr}\left(P_{i} d P_{k} \theta_{h}\right)+\operatorname{tr}\left(P_{i} \theta_{h} P_{k} \theta_{h}\right)\right) .
$$

This splits up into the sum of two terms:

$$
-\frac{i}{4 \pi} \sum_{i \neq k}\left(\log _{z} \lambda_{i}-\log _{z} \lambda_{k}\right)\left(\operatorname{tr}\left(\theta_{h} d P_{k} P_{i}\right)+\operatorname{tr}\left(\theta_{h} P_{i} d P_{k}\right)+\operatorname{tr}\left(P_{i} \theta_{h} P_{k} \theta_{h}\right)\right)
$$

and

$$
-\frac{i}{4 \pi} \sum_{i \neq k}\left(1-\lambda_{i} \lambda_{k}^{-1}\right)\left(\operatorname{tr}\left(\theta_{h} d P_{k} P_{i}\right)+\operatorname{tr}\left(\theta_{h} P_{i} d P_{k}\right)+\operatorname{tr}\left(P_{i} \theta_{h} P_{k} \theta_{h}\right)\right) .
$$

We simplify the term B.1. Using the fact that $\sum_{i} P_{i}=I$ and $\sum_{i} d P_{i}=0$ we have

$$
\sum_{i, k} \log _{z} \lambda_{i}\left(\operatorname{tr}\left(\theta_{h} d P_{k} P_{i}\right)+\operatorname{tr}\left(\theta_{h} P_{i} d P_{k}\right)\right)=0 .
$$

Therefore,

$$
\begin{aligned}
& \sum_{i \neq k} \log _{z} \lambda_{i}\left(\operatorname{tr}\left(\theta_{h} d P_{k} P_{i}\right)+\operatorname{tr}\left(\theta_{h} P_{i} d P_{k}\right)\right) \\
= & -\sum_{i} \log _{z} \lambda_{i}\left(\operatorname{tr}\left(\theta_{h} d P_{i} P_{i}\right)+\operatorname{tr}\left(\theta_{h} P_{i} d P_{i}\right)\right) \\
= & -\sum_{i} \log _{z} \lambda_{i} \operatorname{tr}\left(\theta_{h} d P_{i}\right) .
\end{aligned}
$$

using $d P_{i} P_{i}+P_{i} d P_{i}=d P_{i}$. Similarly we have

$$
\sum_{i \neq k} \log _{z} \lambda_{k}\left(\operatorname{tr}\left(\theta_{h} d P_{k} P_{i}\right)+\operatorname{tr}\left(\theta_{h} P_{i} d P_{k}\right)\right)=\sum_{k} \log _{z} \lambda_{k} \operatorname{tr}\left(\theta_{h} d P_{k}\right) .
$$

For the remaining terms in $B .1$ we have

$$
\sum_{i, k} \log _{z} \lambda_{i} \operatorname{tr}\left(P_{i} \theta_{h} P_{k} \theta_{h}\right)=\sum_{i} \log _{z} \lambda_{i} \operatorname{tr}\left(P_{i} \theta_{h} \theta_{h}\right) .
$$

Hence

$$
\begin{aligned}
\sum_{i \neq k} \log _{z} \lambda_{i} \operatorname{tr}\left(P_{i} \theta_{h} P_{k} \theta_{h}\right) & =\sum_{i} \log _{z} \lambda_{i} \operatorname{tr}\left(P_{i} \theta_{h} \theta_{h}\right)-\sum_{i} \log _{z} \lambda_{i} \operatorname{tr}\left(P_{i} \theta_{h} P_{i} \theta_{h}\right) \\
& =\sum_{i} \log _{z} \lambda_{i} \operatorname{tr}\left(P_{i} \theta_{h} \theta_{h}\right)
\end{aligned}
$$


since $\operatorname{tr}\left(P_{i} \theta_{h} P_{i} \theta_{h}\right)=0$. Similarly we have

$$
\sum_{i \neq k} \log _{z} \lambda_{k} \operatorname{tr}\left(P_{i} \theta_{h} P_{k} \theta_{h}\right)=-\sum_{k} \log _{z} \lambda_{k} \operatorname{tr}\left(P_{k} \theta_{h} \theta_{h}\right) .
$$

Therefore the term (B.1) reduces to

$$
-\frac{i}{2 \pi} \sum_{i} \log _{z} \lambda_{i}\left(\operatorname{tr}\left(P_{i} \theta_{h} \theta_{h}\right)-\operatorname{tr}\left(\theta_{h} d P_{i}\right)\right) .
$$

We compare the term B.3 with $d p_{Y}^{*} \beta$. We have from 5.3.

$$
p_{Y}^{*} \beta=-\frac{i}{2 \pi} \sum_{i} \log _{z} \lambda_{i} \operatorname{tr}\left(\theta_{h} P_{i}\right)
$$

and hence

(B.4) $\quad d p_{Y}^{*}(\beta)=-\frac{i}{2 \pi} \sum_{i} \log _{z} \lambda_{i}\left(\operatorname{tr}\left(-\theta_{h} d P_{i}\right)+\operatorname{tr}\left(\theta_{h} \theta_{h} P_{i}\right)\right)-\frac{i}{2 \pi} \sum_{i} \frac{d \lambda_{i}}{\lambda_{i}} \operatorname{tr}\left(\theta_{h} P_{i}\right)$

Comparing (B.3) and B.4 we obtain the following expression for $\delta\left(p_{Y}^{*}(f)\right)-d p_{Y}^{*}(\beta)$ :

$$
\begin{array}{r}
\delta\left(p_{Y}^{*}(f)\right)-d p_{Y}^{*}(\beta)=-\frac{i}{4 \pi} \sum_{i \neq k}\left(1-\lambda_{i} \lambda_{k}^{-1}\right)\left[\operatorname{tr}\left(\theta_{h} d P_{k} P_{i}\right)+\operatorname{tr}\left(\theta_{h} P_{i} d P_{k}\right)+\operatorname{tr}\left(P_{i} \theta_{h} P_{k} \theta_{h}\right)\right] \\
+\frac{i}{2 \pi} \sum_{i} \frac{d \lambda_{i}}{\lambda_{i}} \operatorname{tr}\left(\theta_{h} P_{i}\right) .
\end{array}
$$

We have, using $\sum_{k} d P_{k}=0$,

$$
\begin{aligned}
& \sum_{i \neq k} \operatorname{tr}\left(\theta_{h} d P_{k} P_{i}\right)+\operatorname{tr}\left(\theta_{h} P_{i} d P_{k}\right) \\
= & -\sum_{i} \operatorname{tr}\left(\theta_{h} d P_{i} P_{i}\right)+\operatorname{tr}\left(\theta_{h} P_{i} d P_{i}\right) \\
= & -\sum_{i} \operatorname{tr}\left(\theta_{h} d P_{i}\right) \\
= & 0 .
\end{aligned}
$$

Similarly, using $\sum P_{k}=I$ and $\operatorname{tr}\left(P_{i} \theta_{h} P_{i} \theta_{h}\right)=0$, we have

$$
\sum_{i \neq k} \operatorname{tr}\left(P_{i} \theta_{h} P_{k} \theta_{h}\right)=\operatorname{tr}\left(\theta_{h} \theta_{h}\right)=0 .
$$

Therefore the expression for $\delta\left(p_{Y}^{*}(f)\right)-d p_{Y}^{*}(\beta)$ in B.5 reduces to

$$
\begin{aligned}
\delta\left(p_{Y}^{*}(f)\right)-d p_{Y}^{*}(\beta)=+\frac{i}{4 \pi} \sum_{i \neq k} \lambda_{i} \lambda_{k}^{-1}\left[\operatorname{tr}\left(\theta_{h} d P_{k} P_{i}\right)+\operatorname{tr}\left(\theta_{h} P_{i} d P_{k}\right)+\operatorname{tr}\left(P_{i} \theta_{h} P_{k} \theta_{h}\right)\right] \\
+\frac{i}{2 \pi} \sum_{i} \frac{d \lambda_{i}}{\lambda_{i}} \operatorname{tr}\left(\theta_{h} P_{i}\right) .
\end{aligned}
$$

For the term

$$
\sum_{i \neq k} \lambda_{i} \lambda_{k}^{-1} \operatorname{tr}\left(P_{i} \theta_{h} P_{k} \theta_{h}\right)
$$

appearing in (B.6) we have, since $\operatorname{tr}\left(P_{i} \theta_{h} P_{i} \theta_{h}\right)=0$,

$$
\begin{aligned}
\sum_{i \neq k} \lambda_{i} \lambda_{k}^{-1} \operatorname{tr}\left(P_{i} \theta_{h} P_{k} \theta_{h}\right) & =\sum_{i, k} \lambda_{i} \lambda_{k}^{-1} \operatorname{tr}\left(P_{i} \theta_{h} P_{k} \theta_{h}\right) \\
& =\operatorname{tr}\left(g \theta_{h} g^{-1} \theta_{h}\right) \\
& =-\operatorname{tr}\left(\theta_{h} \hat{\theta}_{h}\right),
\end{aligned}
$$


where we have set $\hat{\theta}_{h}=g^{-1} \theta_{h} g$. For the term

$$
\sum_{i \neq k} \lambda_{i} \lambda_{k}^{-1}\left[\operatorname{tr}\left(\theta_{h} d P_{k} P_{i}\right)+\operatorname{tr}\left(\theta_{h} P_{i} d P_{k}\right)\right]
$$

we have, using $d P_{i} P_{i}+P_{i} d P_{i}=d P_{i}$ and $\sum_{i} d P_{i}=0$,

$$
\sum_{i \neq k} \lambda_{i} \lambda_{k}^{-1}\left[\operatorname{tr}\left(\theta_{h} d P_{k} P_{i}\right)+\operatorname{tr}\left(\theta_{h} P_{i} d P_{k}\right)\right]=\sum_{k} \lambda_{k}^{-1}\left[\operatorname{tr}\left(\theta_{h} d P_{k} g\right)+\operatorname{tr}\left(\theta_{h} g d P_{k}\right)\right] .
$$

Therefore (B.6) becomes

$$
\begin{aligned}
\delta\left(p_{Y}^{*} f\right)-d p_{Y}^{*} \beta=\frac{i}{4 \pi}\left\{-\operatorname{tr}\left(\theta_{h} \hat{\theta}_{h}\right)+\sum_{k} \lambda_{k}^{-1}\left[\operatorname{tr}\left(\theta_{h} d P_{k} g\right)\right.\right. & \left.+\operatorname{tr}\left(\theta_{h} g d P_{k}\right)\right] \\
& \left.-2 \sum_{k} d \lambda_{k} \lambda_{k}^{-1} \operatorname{tr}\left(\theta_{h} P_{k}\right)\right\} .
\end{aligned}
$$

We have, using $P_{k}^{2}=P_{k}$,

$$
\begin{aligned}
& \sum_{k}\left(\lambda_{k}^{-1} \operatorname{tr}\left(\theta_{h} d P_{k} g\right)-d \lambda_{k} \lambda_{k}^{-1} \operatorname{tr}\left(\theta_{h} P_{k}\right)\right) \\
= & \sum_{k} \lambda_{k}^{-1} \operatorname{tr}\left(\theta_{h} d P_{k} g\right)-\lambda_{k}^{-1} d \lambda_{k} \lambda_{k}^{-1} \operatorname{tr}\left(\theta_{h} P_{k} g\right) \\
= & -\operatorname{tr}\left(\theta_{h} g^{-1} d g\right) \\
= & -\operatorname{tr}\left(\theta_{h} \theta\right),
\end{aligned}
$$

using $d\left(g^{-1}\right)=\sum_{k}\left(\lambda_{k}^{-1} d P_{k}-\lambda_{k}^{-1} d \lambda_{k} \lambda_{k}^{-1} P_{k}\right)$, where we have set $\theta=g^{-1} d g$. Similarly,

$$
\sum_{k}\left(\lambda_{k}^{-1} \operatorname{tr}\left(\theta_{h} g d P_{k}\right)-d \lambda_{k} \lambda_{k}^{-1} \operatorname{tr}\left(\theta_{h} P_{k}\right)\right)=-\operatorname{tr}\left(\hat{\theta}_{h} \theta\right) .
$$

Hence

$$
\delta\left(p_{Y}^{*}(f)\right)-d p_{Y}^{*}(\beta)=p_{Y}^{*} \pi^{*}\left(\frac{i}{4 \pi}\left(\operatorname{tr}\left(\hat{\theta}_{h} \theta_{h}\right)+\operatorname{tr}\left(\theta \theta_{h}\right)+\operatorname{tr}\left(\theta \hat{\theta}_{h}\right)\right)\right) .
$$

\section{REFERENCES}

[1] J. Baez and A. Lauda, Higher-dimensional algebra, V: 2-groups, Theory Appl. Categ. 12:423-491, 2004.

[2] J. Baez, D. Stevenson, A. Crans, and U. Schreiber, From loop groups to 2-groups, Homology, Homotopy Appl., 9(2):101-135, 2007.

[3] O. Ben-Bassat, Equivariant gerbes on complex tori, J. Geom. Phys., 64:209-221, 2013.

[4] R. Bott, Lectures on characteristic classes and foliations, Notes by Lawrence Conlon, with two appendices by J. Stasheff. Lectures on algebraic and differential topology (Second Latin American School in Math., Mexico City, 1971), pp. 1-94. Lecture Notes in Mathematics, Vol. 279. Springer, Berlin, 1972.

[5] P. Bouwknegt, A.L. Carey, V. Mathai, M.K. Murray and D. Stevenson, Twisted $K$-theory and K-theory of bundle gerbes, Comm. Math. Phys., 228, 2002, 17-49.

[6] J.-L. Brylinski, Loop spaces, characteristic classes and geometric quantization, Progress in Mathematics, 107, Birkhäuser Boston, Inc., Boston, MA, 1993.

[7] J.-L. Brylinski, Gerbes on complex reductive Lie groups, preprint arXiv:math/0002158.

[8] J.-L. Brylinski and D. A. McLaughlin, The geometry of degree-four characteristic classes and of line bundles on loop spaces, I, Duke Math. J. 75(3):603-638, 1994.

[9] A.L. Carey, J. Mickelsson and M.K. Murray, Index theory, gerbes and Hamiltonian quantisation, Comm. Math. Phys., 183, 1997, 717-722.

[10] A.L. Carey, J. Mickelsson and M.K. Murray, Bundle gerbes applied to quantum field theory, Reviews in Mathematical Physics, 12(1), 2000, 65-90.

[11] A.L. Carey, S. Johnson, M.K. Murray, D. Stevenson, and B-L. Wang, Bundle gerbes for ChernSimons and Wess-Zumino-Witten theories, Comm. Math. Phys., 259(3):577-613, 2005.

[12] D. Carpentier, P. Delplace, M. Fruchart, K. Gawedzki and C. Tauber, Construction and properties of a topological index for periodically driven time-reversal invariant 2D crystals, Nuclear Physics $B, 896,779-834,2015$. 
[13] D. Chatterjee, On Gerbs, PhD thesis, Cambridge, 1998.

[14] J.L. Dupont, Curvature and characteristic classes, Lecture Notes in Mathematics, Vol. 640. Springer, Berlin, 1978.

[15] J. Duskin, Simplicial matrices and the nerves of weak $n$-categories I: nerves of bicategories, Theory Appl. Categ., 9(10):198-308, 2002.

[16] E.M. Friedlander, Etale homotopy of simplicial schemes, Annals of Math. Studies Vol. 104, Princeton University Press, Princeton, NJ, 1982.

[17] N. Ganter, Categorical tori, arXiv:1406.7046.

[18] K. Gawedzki, Topological actions in two-dimensional quantum field theories. in 'Nonperturbative quantum field theory' edited by G. 't Hooft et al., (Cargese, 1987), 101-141, NATO Adv. Sci. Inst. Ser. B: Phys., 185, New York : Plenum Press, 1988.s

[19] K. Gawedzki, Bundle gerbes for topological insulators, arXiv:1512.01028.

[20] P. Goerss and J. Jardine Simplicial homotopy theory, Progress in Mathematics, 174. Birkhäuser Verlag, Basel, 1999. xvi+510 pp.

[21] K. Gomi, Relationship between equivariant gerbes and gerbes over the quotient space, Commun. Contemp. Math. 7(2):207-226, 2005.

[22] K. Gomi, Reduction of strongly equivariant bundle gerbes with connection and curving, arXiv:math/0406144.

[23] Pedram Hekmati, Michael K. Murray, Danny Stevenson and Raymond F. Vozzo, The FaddeevMickelsson-Shatashvili anomaly and lifting bundle gerbes, Comm. Math. Phys., 319(2), (2013), 379-393.

[24] P. Hořava, Type IIA D-branes, K-theory and Matrix Theory, Adv. Theor. Math. Phys., 2 (1999) 1373.

[25] A. Kapustin, D-branes in a topologically non-trivial B-field, Adv. Theor. Math. Phys., 4 (2001) 127.

[26] T.P. Killingback, World-sheet anomalies and loop geometry, Nuclear Phys. B 288, no. 3-4, 578-588, 1987.

[27] V. Mathai and D. Stevenson. Chern character in twisted $K$-theory: equivariant and holomorphic cases, Comm. Math. Phys., 236(1):161-186, 2003.

[28] E. Meinrenken, The basic gerbe over a compact simple Lie group, Enseign. Math. (2), 49(3-4):307$333,2003$.

[29] R. Mickler, A New Look at Chern-Simons on Circle Bundles I: The Caloron Correspondence, arXiv: 1507.01626.

[30] R. Minasian and G. Moore, K-theory and Ramond-Ramond charge, J. High Energy Phys., 05 (2000) 02

[31] G. Moore and E. Witten, Self-duality, Ramond-Ramond fields and K-theory, J. High Energy Phys., 05 (2000) 032.

[32] M.K. Murray, Bundle gerbes, J. London Math. Soc. (2), 54(2):403-416, 1996.

[33] M.K. Murray and D. Stevenson, Higgs fields, bundle gerbes and string structures, Comm. Math. Phys., 243(3):541-555, 2003.

[34] M.K. Murray and D. Stevenson, The basic bundle gerbe on unitary groups, J. Geom. Phys., 58(11):1571-1590, 2008.

[35] T. Nikolaus and C. Schweigert, Equivariance in higher geometry, Adv. Math. 226(4):3367-3408. 2011.

[36] D. Stevenson, The geometry of bundle gerbes, PhD Thesis, University of Adelaide, 2000, arXiv: math.DG/0004177.

[37] D. Stevenson, Bundle 2-gerbes, Proc. Lond. Math. Soc. 88(3):405-435, 2004.

[38] M. Stienon, Equivariant Dixmier-Douady classes, Math. Res. Lett. 17(1):127-145, 2010.

[39] S. Stolz and P. Teichner, What is an elliptic object? Topology, geometry and quantum field theory, 247-343, London Math. Soc. Lecture Note Ser., 308, Cambridge Univ. Press, Cambridge, 2004.

[40] R. Street, The algebra of oriented simplexes, J. Pure Appl. Algebra, 49(3):283-335, 1987.

[41] K. Waldorf, String connections and Chern-Simons theory, Trans. Amer. Math. Soc. 365(3):4393$4432,2013$.

[42] E. Witten, Global anomalies in string theory. Symposium on anomalies, geometry, topology (Chicago, Ill., 1985), 61-99, World Sci. Publishing, Singapore, 1985.

[43] E. Witten, The index of the Dirac operator in loop space, Elliptic curves and modular forms in algebraic topology 161-181, 1988. 
(Michael Murray) School of Mathematical Sciences, University of Adelaide, Adelaide, SA 5005, Australia

E-mail address: michael.murray@adelaide.edu.au

(David M. Roberts) School of Mathematical Sciences, University of Adelaide, Adelaide, SA 5005, Australia

E-mail address: david.roberts@adelaide.edu.au

(Danny Stevenson) School of Mathematical Sciences, University of Adelaide, Adelaide, SA 5005, Australia

E-mail address: daniel.stevenson@adelaide.edu.au

(Raymond Vozzo) School of Mathematical Sciences, University of Adelaide, Adelaide, SA 5005, Australia

E-mail address: raymond.vozzo@adelaide.edu.au 